\title{
Complexity and boost symmetry
}

\author{
Ying Zhao* \\ Stanford Institute for Theoretical Physics and Department of Physics, Stanford University, \\ Stanford, California 94305-4060, USA
}

(Received 15 July 2018; published 10 October 2018)

\begin{abstract}
We find that the time dependence of holographic complexity is controlled by the Rindler boost symmetry across the horizon. By studying the collision energy experienced by an infalling object, we see that the breaking of this boost symmetry is closely related to firewalls, which in turn shows the connection between the time dependence of complexity and firewalls. We further identify the black and white hole interiors as two tapes storing different parts of the minimal circuit preparing the state. Depending on whether the quantum gates are being laid on the tape at a particular moment, each tape can be in two states: working, or locked. We interpret the existence of firewalls as the locking of tapes.
\end{abstract}

DOI: 10.1103/PhysRevD.98.086011

\section{INTRODUCTION}

The growth of the black hole interior is a mystery. From the field theory point of view, thermal equilibrium is quickly achieved, but from the bulk geometry, one can see that the interior keeps expanding. It is conjectured that this reflects the increase of the state complexity-i.e., the minimal number of simple gates needed to prepare a state starting from some simple reference state [1].

The picture of a tensor network lying in spacetime $[2,3]$ offers an intuitive explanation about the duality between boundary state complexity and bulk spacetime. It also makes explicit the idea of a black hole interior as emergent spacetime [4-6]. We can view the tensor network as a quantum circuit lying inside the horizon, recording the dynamics that creates the state starting from some simple state. The complexity is one important parameter characterizing this dynamics. In particular, if the state is made by inserting various precursors to the thermofield double, complexity keeps track of the dynamics of the conformal field theory (CFT) disturbed at various points.

In Refs. [5,7], the complexity of a holographic state is conjectured to be dual to the volume of maximal surface anchored at the boundary. In Refs. [6,8], it is pointed out that the action in the Wheeler-DeWitt (WDW) patch offers a better candidate for complexity. Both prescriptions give certain features of the time dependence of complexity, like linear increase for subexponential time, switchback

*zhaoying@stanford.edu

Published by the American Physical Society under the terms of the Creative Commons Attribution 4.0 International license. Further distribution of this work must maintain attribution to the author(s) and the published article's title, journal citation, and DOI. Funded by SCOAP. effect, etc., which match quite well with what one expects from the quantum circuit picture.

We shall explore the origin of these various features and see that the time dependence of complexity is basically a consequence of boost symmetry across the horizon, as indicated in Fig. 1. Notice that the Schwarzschild time inside the horizon is synchronized with the Schwarzschild time outside. This boost symmetry is the origin of the linear time dependence of complexity. With the insertion of various precursors, the uniform dynamics is disrupted, and the bulk symmetry is broken. However, it is broken in a particularly simple pattern which results from the Rindler-like nature of near horizon geometry. We will see that events near the horizon can play a crucial role. If they affect the synchronization, there will be abnormal time dependence of complexity, and vice versa. ${ }^{1}$

The quantum circuit description of the black hole interior was given by Hartman and Maldacena [4]. The idea of a tape storing the quantum circuit behind the future horizon appeared in Ref. [9]. We will see that in order to incorporate the possibility of complexity decrease and the existence of firewalls [10], we need two tapes: future and past tapes, which are stored in the future and past interiors, respectively [11]. There is a family of examples of black holes in which the minimal quantum circuit preparing the state is explicitly known and is composed of segments of forward and backward Hamiltonian evolutions $[12,13]$. The forward Hamiltonian evolution part is stored in the future tape, while the backward evolution part is stored in the past tape. When the quantum gates preparing the state are laid on one particular tape, we say that the tape is working, and the

\footnotetext{
${ }^{1}$ In this thesis, we are talking about subexponential time. We do not really know what will happen after the complexity saturates.
} 


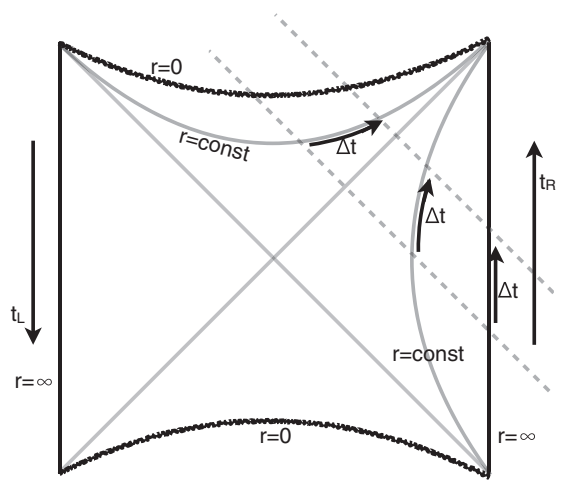

FIG. 1. Boost symmetry across the horizon. The Schwarzschild time inside the horizon synchronizes with the Schwarzschild time outside.

corresponding horizon is smooth. When there are no gates laid down on that tape, the entry to the interior is forbidden. From the point of view of an exterior observer, a black hole is just a particle with certain properties [14]. What is special about it is that there is spacetime behind the horizon, which an infalling observer can enter. In that sense, the horizon is like a door to the interior region. One learns that whether the door is open or not depends on the working status of the corresponding tape; one can cross the horizon only when the corresponding tape is working.

In this paper, we shall explore the time dependence of the complexity using the following assumptions: ${ }^{2}$

(1) In holographic duality, the complexity of a boundary state is dual to some bulk geometric quantity inside the Wheeler-DeWitt patch.

(2) Tensor networks support spacetime. They have locality down to the AdS scale, and respect the symmetry of spacetime-in particular, time translation symmetry and rotational symmetry.

(3) Complexity counts the number of gates in the tensor network.

(4) Contributions to complexity from very small regions are also small.

The most important assumption we use throughout this paper is probably the validity of classical general relativity, at least before very late times.

By performing a more detailed matching between the picture of the quantum circuit preparing a state and the dual black hole geometry, we then identify the different roles played by future and past horizons. In the minimal circuit preparing the state, there can be both forward and backward Hamiltonian evolutions. They are stored in two different tapes. The future interior serves as the future tape, storing the forward Hamiltonian evolution, while the past interior is the past tape, storing the backward Hamiltonian evolution.

\footnotetext{
${ }^{2}$ In these four assumptions, assumption 2 essentially implies assumptions 1, 3, and 4. I thank Daniel Harlow for pointing this out.
}

In this sense, the existence of past horizons means that complexity can increase towards the past, and this is a manifestation of time reversibility.

Here we should also emphasize the limit of this picture. The examples we studied are special. We know the minimal circuit preparing them, and their minimal circuits are made of Hamiltonian evolutions perturbed at various points. We do not know what will happen for more general states.

This paper is organized as follows: In Sec. II we give a brief review of some known results about holographic complexity, and then illustrate the idea of symmetry determining complexity by explicit calculations in shockwave geometries. In particular, without a detailed prescription, we will recover the earlier results about the time dependence of complexity in Refs. [5-7] from the above assumptions. In Sec. II D, we use this idea to show the connection between the time dependence of complexity and the smoothness of horizons [10]. We study the connection between the boost symmetry breaking and collision energy in general relativity. In Sec. III, we introduce the two-tape picture and make a connection between the working status of the tapes and the smoothness of the horizon.

For the convenience of the reader, we shall list some conventions used throughout this section:

(1) $D$ refers to the spacetime dimension of the bulk.

(2) Our convention of time will be that time flows upward on the right side of a Penrose diagram, and downward on the left side; see Fig. 1. So a precursor $W_{L}\left(t_{w}\right)=e^{i H_{L} t_{w}} W_{L} e^{-i H_{L} t_{w}}$ acting on the left CFT from the remote past would have large positive $t_{w}$.

(3) We will use boost symmetry and time translation symmetry interchangeably.

\section{TIME DEPENDENCE OF COMPLEXITY AND BOOST SYMMETRY}

\section{A. Known results about holographic complexity}

Given a set of simple gates and a simple reference state, the complexity of a state is defined as the minimum number of gates needed to prepare the state. For a continuum field theory, we still do not have a rigorous definition of complexity, but we expect there exists a similar quantity which characterizes how hard it is to prepare a state starting from a particular simple reference state (a thermofield double for a two-sided black hole). On the bulk side, it was proposed that complexity is dual to the volume of the maximal surface anchored on the boundary, with some prefactor involving the cosmological constant $[5,7]$. Later, it was proposed that complexity equals the action of the Wheeler-DeWitt patch in the bulk [6,8], and a universal rate of complexity increase was found. Both proposals were tested in various examples. 
For example, take the time evolution of the thermofield double:

$$
\left|\psi\left(t_{R}, t_{L}\right)\right\rangle=\frac{1}{\sqrt{Z}} \sum_{n} e^{-\frac{\beta}{2} E_{n}}|n\rangle_{L}|n\rangle_{R} e^{-i E_{n}\left(t_{R}-t_{L}\right)},
$$

whose holographic dual is a two-sided black hole in anti-de Sitter space [15] (Fig. 1). Since black hole dynamics is chaotic, we do not expect shortcuts (fast forwarding in Ref. [16]) before an exponentially long time. The complexity will increase linearly with time after an initial transient period of the order of the thermal time [6,7]:

$$
\mathcal{C}\left(t_{R}, t_{L}\right)=\mathcal{C}(|\mathrm{TFD}\rangle)+C\left|t_{R}-t_{L}\right|
$$

We can also consider more complicated states by perturbing the system at various times. If we act on the state with a precursor $W_{L}\left(t_{w}\right)=e^{i H_{L} t_{w}} W_{L} e^{-i H_{L} t_{w}}$, where $W_{L}$ is some thermal scale perturbation, it amounts to throwing in a thermal quantum at early time $t_{w}$. In the dual geometry,

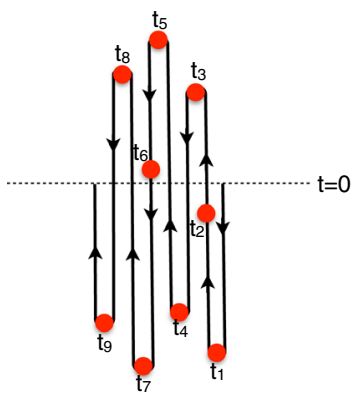

FIG. 2. Product of multiple precursors.

there is a shockwave lying close to the horizon [12]. What is the effect of such a perturbation? For a black hole of size $l_{\text {AdS }}$, we expect that this quantum takes a scrambling time $t_{*}=\frac{\beta}{2 \pi} \log S$ to affect the entire system, during which the perturbation has little effect on complexity. After that, the complexity will increase linearly. Bulk calculations indeed give $[6,7]$

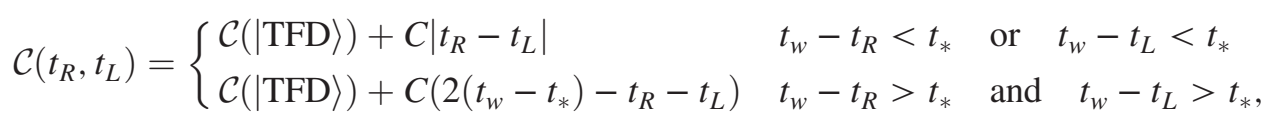

where in the first line it behaves the same as the unperturbed system. The subtraction of $2 t_{*}$ in the second line is called the switchback effect $[7,17,18]$. The switchback effect is due to cancellations between forward and backward time evolutions during which the perturbation has not yet affected much of the system. Also, as was pointed out in Refs. [7,10], in the range $t_{R}<t_{w}-t_{*}, t_{L}<t_{w}-t_{*}$, the complexity always decreases with $t_{R}$. In contrast, without any perturbations the complexity will increase with $t_{R}$ as long as $t_{R}>t_{L}$. This is what we mean by abnormal time dependence. We will use this to diagnose the transparency of horizons in Sec. II D [10].

We can also consider the insertion of multiple precursors:

$$
\begin{aligned}
& W_{n}\left(t_{n}\right) W_{n-1}\left(t_{n-1}\right) \ldots W_{2}\left(t_{2}\right) W_{1}\left(t_{1}\right) \\
& =e^{-i H t_{n}} W_{n} e^{i H\left(t_{n}-t_{n-1}\right)} W_{n-1} e^{i H\left(t_{n-1}-t_{n-2}\right)} \\
& \quad \ldots W_{2} e^{i H\left(t_{2}-t_{1}\right)} W_{1} e^{i H t_{1}}
\end{aligned}
$$

where the perturbations are well separated, $\left|t_{i}-t_{i+1}\right|>2 t_{*}$, so that the spreadings of different precursors do not interfere. See Fig. 2. The arrows indicate the direction of time evolution.

From considerations of quantum circuits, we expect one switchback from each turn, so the complexity of this operator will be

$$
\mathcal{C}=C\left(t_{f}-2 n_{s b} t_{*}\right),
$$

where $t_{f}$ is total folded time, and $n_{s b}$ is the number of switchbacks. On the gravity side, the system is dual to black hole geometries perturbed by multiple shockwaves separated by large times [13]. The result (2.4) is reproduced in gravity calculations $[6,7]$.

In previous examples, we considered systems dual to black holes of size $l_{\text {Ads }}$. We can also consider a bigger system with position-dependent perturbations originating at position $x_{w}$. Now the perturbation also spreads ballistically. In Refs. [5,19,20], geometries with localized shocks were studied in detail, and it was shown that in the complexity there will be more complicated position-dependent cancellations [5]. Roughly speaking, the perturbation spreads with butterfly velocity $v_{B}$, so the degrees of freedom at position $x$ feel the effect of the perturbation at time $t_{w}-\frac{\left|x-x_{w}\right|}{v_{B}}$. This means that the contribution to complexity from site $x$ will be proportional to $2\left(t_{w}-\frac{\left|x-x_{w}\right|}{v_{B}}-t_{*}\right)$.

In all the above calculations, the time dependence of complexity shows certain robust features, like linear increase at large times, a switchback delay time of $2 t_{*}$, and abnormal decrease when the interior contains shockwaves. In the rest of this section, we will rederive these results just from general assumptions 1 to 4 . We will see that, in the bulk, these features are essentially consequences of symmetry and a particular pattern of symmetry breaking which results from the Rindler nature of horizons. 


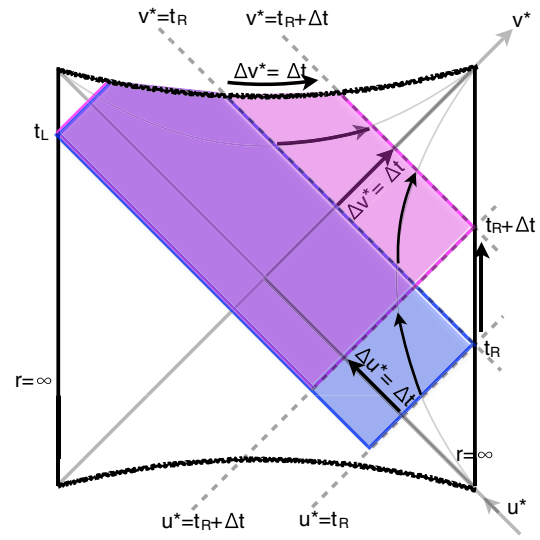

(a) Two WDW patches at different $t_{R}$ with $t_{L}$ fixed.

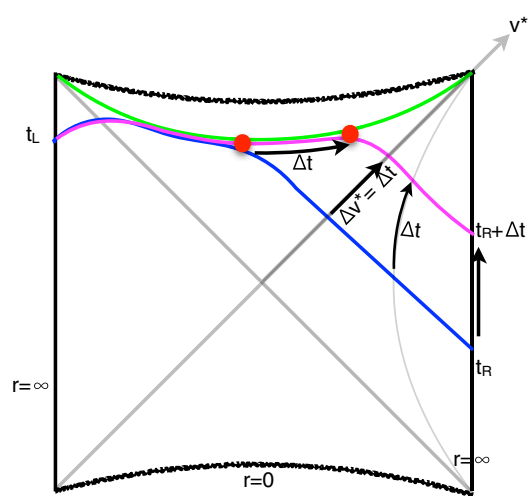

(b) Two maximal surfaces at different $t_{R}$ with $t_{L}$ fixed.

FIG. 3. Bulk duals of $\left|\psi\left(t_{R}, t_{L}\right)\right\rangle$ and $\left|\psi\left(t_{R}+\Delta t, t_{L}\right)\right\rangle$.

\section{B. Linear increase of complexity: Boost symmetry across the horizon}

Let us first look at the time evolution of the thermofield double without perturbations [Eq. (2.1)]. The state $\left|\psi\left(t_{R}, t_{L}\right)\right\rangle$ describes the Wheeler-DeWitt (WDW) patch at time $\left(t_{R}, t_{L}\right)$-i.e., the bulk region that is spacelike separated from the chosen boundary time [21]. From assumption 1, complexity is dual to a certain geometric quantity defined within the WDW patch.

Earlier, we mentioned boost symmetry across the horizon. Here we shall make this statement precise. In Fig. 1, if $t_{R}$ increases by $\Delta t$, the right boundary of the WDW patch will move by $\Delta t$ along any $r=$ constant curve. This is more easily seen in Eddington-Finkelstein coordinates: We define the ingoing time as $d v^{*}=d t+\frac{d r}{f(r)}$. Then the metric becomes

$$
d s^{2}=-f(r) d v^{* 2}+2 d r d v^{*}+r^{2} d \Omega_{D-2}^{2}
$$

These coordinates cover the right exterior and future interior regions in the Penrose diagram, and $v^{*}$ is a good time coordinate on the right future horizon. The boost symmetry in these two quadrants can now be written as

$$
v^{*} \rightarrow v^{*}+\Delta t .
$$

We can similarly define $d u^{*}=d t-\frac{d r}{f(r)}$, and it covers the right exterior region as well as the white hole interior.

We can intuitively view $v^{*}$ as a trajectory of a light beam sent from the boundary at time $t_{R}$ along radial directions. The beam will stay on the boundary of the WDW patch $v^{*}=t_{R}$. As $t_{R}$ increases, the spacetime region between $v^{*}=t_{R}$ and $v^{*}=t_{R}+\Delta t$ is scanned by the beam, and the time dependence of complexity is seen in this scan. As we will make precise later, the scan can tell us whether the interior is growing uniformly, which shows whether the dynamics of making the state is going well without being disrupted. We should emphasize here that the scanning beam is merely a geometric analogy. All we want is something representing the boundary of the WDW patch. We do not mean physically throwing in any quanta.

Also note that, even though we work with a thermofield double as an example, what we mean by boost symmetry in this paper is a property near the horizon and does not rely on the existence of the other side.

Let us fix $t_{L}$ and just evolve $t_{R}$. When $t_{R}-t_{L}$ is large, we see a pattern emerge.

Figure 3(a) shows WDW patches at two times. As we increase $t_{R}$, we move the upper-right boundary of the WDW patch from $v^{*}=t_{R}$ to $v^{*}=t_{R}+\Delta t$, and the lowerright boundary from $u^{*}=t_{R}$ to $u^{*}=t_{R}+\Delta t$. The black hole interior is expanding, while the white hole interior is shrinking. With large $t_{R}-t_{L}$, the white hole interior is small, and we expect it to have less and less effect on the complexity (assumption 4). The change in the WDW patch is basically to add a piece of Fig. 4(a) to the interior region. Regions like this will be the basic building blocks for our following discussions. They have the feature of being

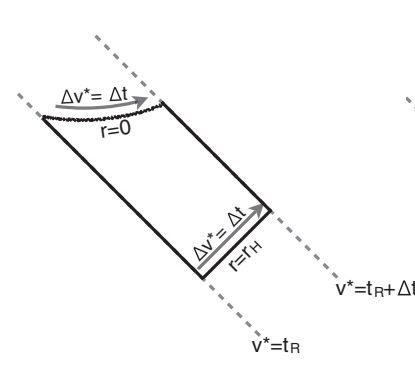

(a)

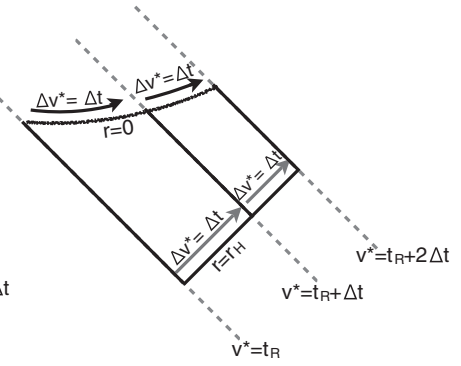

(b)
FIG. 4. Increase of WDW patch as right time increases from $t_{R}$ to $t_{R}+\Delta t$. 
uniform in the Schwarzschild time direction. Look at Fig. 4(b). We have divided it into two parts. The two parts are related by a symmetry transformation $v^{*} \rightarrow v^{*}+\Delta t$, so we expect them to give equal contributions to complexity. As a consequence, the contribution of a region like this to complexity will be proportional to $\Delta v^{*}{ }^{3}$ From now on, we will denote it by $C \Delta v^{*}$ :

$$
\Delta \mathcal{C}=C \Delta v^{*}
$$

This symmetry is the reason for which we find a linear increase of complexity. But of course, without a specific prescription, we cannot fix the coefficient $C$.

The above argument does not depend on the detailed prescriptions of the bulk dual of complexity. It only requires that the prescription respect time translation symmetry; i.e., we do not use different prescriptions at different times. For example, we draw maximal-volume surfaces in Fig. 3(b). The maximal-volume surfaces hug the limiting surface (green line) until they separate (red dots). The volume increases linearly with the separation time, and that time is synchronized (up to an additive constant) with boundary time $t_{R}$ as a consequence of the above mentioned symmetry.

There will be a transient period of the order of the thermal time, during which the above symmetry argument does not apply. It happens when the tip of the WDW wedge leaves the singularity and the corresponding piece of the interior starts to shrink. We do not expect this period to give big contributions to complexity. Also, we are interested in time scales much bigger than the thermal time, so we will ignore this transient. The transient period ends when the shrinking interior piece becomes very small, or the maximal surface touches the limiting surface. After that, the robust linear increase kicks in, and the corrections continue to die off exponentially. Symmetry dictates the linear time dependence in Eq. (2.2).

\section{Breaking boost symmetry: Abnormal time dependence of complexity}

What if we perturb the state by inserting a precursor $W_{L}\left(t_{w}\right)=e^{i H t_{w}} W_{L} e^{-i H t_{w}}$ with $t_{w}$ large? The geometry was studied in Ref. [12]. Now there is a shockwave lying close to the horizon, and it would break the boost symmetry in a specific pattern; see Fig. 5.

From earlier discussions, we see that what determines the time dependence of complexity is how the interior portion of the WDW patch changes as we vary the boundary time-i.e., as the detecting beam scans the interior, it would keep a record of what is going on. Now, with a shockwave present, the scanning beam will be pushed forward by an amount depending on the position

\footnotetext{
${ }^{3}$ In complexity-action prescription, additivity of action is a subtle issue, which was discussed in Ref. [22].
}

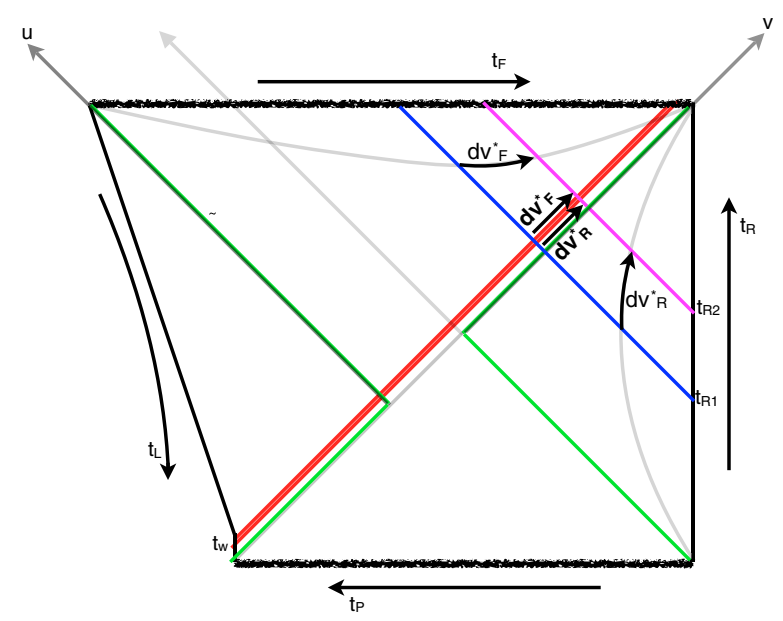

FIG. 5. Two-sided black hole with one spherically symmetric shockwave.

where the beam meets the shockwave. Mathematically, $\partial_{v^{*}}$ is no longer a Killing vector. The time separation $\Delta v^{*}$ between the two beams inside the horizon will no longer be the same as that outside. In Fig. 5, we consider two ingoing null geodesics from the right boundary with time separation $\Delta t_{R}=\Delta v_{R}^{*}$. Across the shockwave, there will be relative time dilation, and $\Delta v^{*}$ will jump when the two geodesics pass the shell.

To quantify this effect, note that near the horizons, the geometry is Rindler-like on both sides of the shockwave:

$$
d s^{2}=-f(r) d v^{* 2}+2 d r d v^{*}+r^{2} d \Omega_{D-2}^{2},
$$

where $f(r)=\frac{4 \pi}{\beta}\left(r-r_{H}\right)$. Across the shell, there is discontinuity in the $v^{*}$ coordinate:

$$
\frac{d v_{F}^{*}}{d v_{R}^{*}}\left(t_{R}\right)=\frac{f\left(r_{w}\right)}{\tilde{f}\left(r_{w}\right)}=\frac{r_{H}-r_{w}}{\tilde{r}_{H}-r_{w}}=\frac{r_{H}-r_{w}}{\delta r_{H}+r_{H}-r_{w}},
$$

where $r_{w}$ is the radius at which the ingoing null line crosses the shockwave, and $\delta r_{H}$ is the increase of the horizon radius due to the extra quantum. If we throw in a thermal scale quantum, we have

$$
\delta S \sim 1, \quad \frac{\delta r_{H}}{r_{H}}=\frac{1}{D-2} \frac{\delta S}{S} .
$$

Rindler geometry also gives

$$
r_{w}-r_{H}=-\frac{\beta}{4 \pi} e^{-\frac{2 \pi}{\beta}\left(t_{w}-t_{R}-2 R^{*}\right)},
$$

where $\frac{R^{*}}{\beta}$ is an order-one constant depending on the asymptotic geometry and dimension. So, we see that the dilation factor is given by 


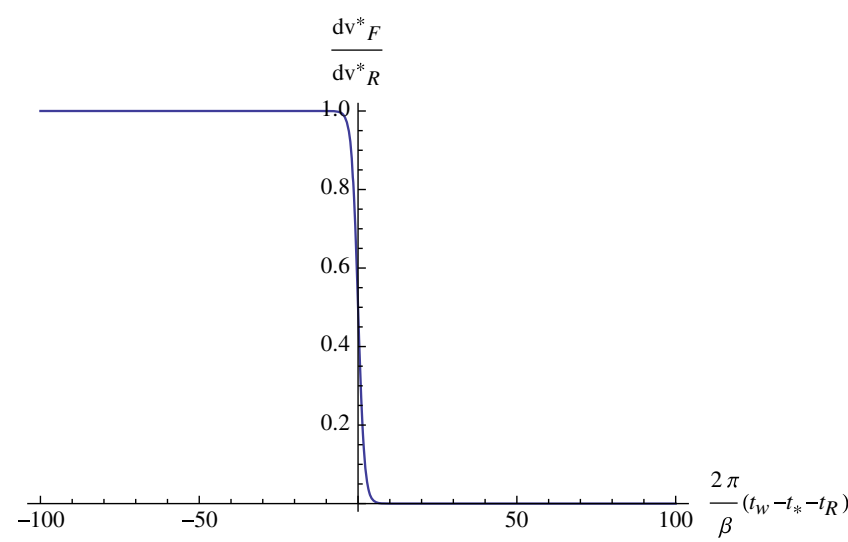

FIG. 6. Eddington-Finkelstein time dilation factor as we go across the shockwave, as a function of boundary time.

$$
\frac{d v_{F}^{*}}{d v_{R}^{*}}\left(t_{R}\right)=\frac{1}{1+c e^{\frac{2 \pi}{\beta}\left(t_{w}-t_{R}-t_{*}\right)}},
$$

where $c=\frac{1}{D-2} \frac{4 \pi r_{H}}{\beta} e^{-\frac{4 \pi}{\beta} R^{*}} \delta S$ is an order-one constant.

This time dilation factor quantitatively characterizes to what extent the boost symmetry is broken. Note that to arrive at this factor in Eq. (2.8), we only use the Rindlerlike geometry of the horizon. Its functional form is completely robust. Different dimensions or different asymptotic boundary conditions only change the constant $c$. Let us look at the behavior of this function (Fig. 6). When $t_{w}-t_{R}<t_{*}, d v_{F}^{*}=d v_{R}^{*}$; i.e., there is still good symmetry across the horizon. But when $t_{w}-t_{R}>t_{*}$, $d v_{F}^{*}=e^{-\frac{2 \pi}{\beta}\left(t_{w}-t_{*}-t_{R}\right)} d v_{R}^{*}$ is exponentially smaller than $d v_{R}^{*}$; i.e., as we increase $t_{R}$, the future interior does not grow accordingly. We have seen that the linear increase of complexity is a result of the steady expansion of the black hole interior. Now this symmetry is broken, and it gives rise to abnormal time dependence of complexity. ${ }^{4}$ From here we already see that the time dependence of complexity is closely related to the transparency of the horizon, as proposed by Susskind in Ref. [10]. We will explore this more in Secs. II D and III.

Because the perturbation at time $t_{w}$ has very low energy, it causes very little change to the system right after the perturbation. In order to explore the time dependence of complexity, let us begin with a boundary time $t_{L}$ right above $t_{w}$. (In our convention, this means that $t_{L}$ is slightly smaller than $t_{w}$.) Let $t_{R}=t_{L}=t_{w}$. In Fig. 7(a), the dual WDW patch is shown in blue.

\footnotetext{
${ }^{4}$ In the type of perturbation we considered here (low energy, early time), the symmetry is broken in a particularly simple pattern. The only effect of the shockwave is to give a relative time dilation across the horizon. The change of interior always has the same shape as in Fig. 4. The only difference is that $\Delta v^{*}$ inside horizon can be smaller than $\Delta v^{*}$ outside, which will give rise to a shift in the time dependence of complexity.
}

Next, let us push $t_{L}$ upward with $t_{R}$ fixed [Fig. 7(b)]; i.e., we decrease $t_{L}$ until $t_{w}-t_{L}>t_{*}$ [Fig. 7(c)]. The change in the white hole interior will only show up as some transient behavior for thermal time, so we ignore it. The black hole interior steadily expands. We have

$$
\begin{aligned}
\Delta \mathcal{C}_{1} & =C \Delta u_{F}^{*}, \\
\frac{d \mathcal{C}_{1}\left(t_{L}, t_{R}=t_{w}\right)}{d t_{L}} & =C \frac{d u_{F}^{*}\left(t_{L}\right)}{d t_{L}}=-C, \\
\mathcal{C}_{1}\left(t_{L}, t_{R}=t_{w}\right) & =\mathcal{C}(|\mathrm{TFD}\rangle)+C\left(t_{w}-t_{L}\right) .
\end{aligned}
$$

Thus, we see that the complexity increases as we push $t_{L}$ upward.

Now, we fix the left time at $t_{L}$, and move $t_{R}$ downward; see Fig. 8.

We start from Fig. 8(a), where the complexity is given by Eq. (2.9). With $t_{w}-t_{R}<t_{*}$ [Fig. 8(b)], we can still ignore the white hole interior. The black hole interior is shrinking, but here we encounter the shockwave, so we need to take into account the time dilation according to Eq. (2.8).

$$
\begin{aligned}
\Delta \mathcal{C}_{1}= & C \Delta v_{F}^{*}, \quad d v_{R}^{*}=d t_{R}, \\
\frac{d \mathcal{C}_{1}\left(t_{L}, t_{R}\right)}{d t_{R}}= & C \frac{d v_{F}^{*}}{d v_{R}^{*}}\left(t_{R}\right)=\frac{C}{1+c e^{\frac{22}{\beta}\left(t_{w}-t_{*}-t_{R}\right)}} \\
\mathcal{C}_{1}\left(t_{L}, t_{R}\right)= & \mathcal{C}_{1}\left(t_{L}, t_{w}\right) \\
& +C\left(t_{R}+\frac{\beta}{2 \pi} \log \left(1+c e^{\frac{2 \pi}{\beta}\left(t_{w}-t_{*}-t_{R}\right)}\right)\right)-C t_{w} \\
= & \mathcal{C}(|\mathrm{TFD}\rangle)+C\left(t_{R}-t_{L}\right) .
\end{aligned}
$$

In this regime $\left[t_{w}-t_{R}<t_{*}\right.$, Fig. 8(c)], the symmetry across the horizon is still approximately satisfied, and the complexity increases linearly with the right boundary time. So far we have not encountered any abnormal behaviors of complexity.

Next, let us push $t_{R}$ down further; i.e., consider when $t_{L}<t_{w}-t_{*}$ and $t_{R}<t_{w}-t_{*}$. See Fig. 9 .

Now, the interiors of both the black hole and the white hole change [Fig. 9(b)]. We need to consider the contributions to the complexity from both regions. Here, when we talk about the contributions to the complexity from different regions, we are implicitly using assumption 2 , that the tensor network has locality down to AdS scale. We have

$$
\begin{aligned}
\Delta \mathcal{C}_{1}= & C \Delta v_{F}^{*}-C \Delta u_{P}^{*}, \\
\frac{d \mathcal{C}_{1}\left(t_{L}, t_{R}\right)}{d t_{R}}= & C\left(\frac{d v_{F}^{*}}{d v_{R}^{*}}\left(t_{R}\right)-\frac{d u_{P}^{*}}{d t_{R}}\right)=-C \frac{c e^{\frac{2 \pi}{\beta}\left(t_{w}-t_{*}-t_{R}\right)}}{1+c e^{\frac{2 \pi}{\beta}\left(t_{w}-t_{*}-t_{R}\right)}}, \\
\mathcal{C}_{1}\left(t_{L}, t_{R}\right)= & \mathcal{C}_{1}\left(t_{L}, t_{w}-t_{*}\right) \\
& +C \frac{\beta}{2 \pi} \log \left(\frac{1+c e^{\frac{2 \pi}{\beta}\left(t_{w}-t_{*}-t_{R}\right)}}{1+c e^{\frac{2 \pi}{\beta}\left(t_{w}-t_{*}-\left(t_{w}-t_{*}\right)\right)}}\right), \\
= & \mathcal{C}(|\mathrm{TFD}\rangle)+C\left(2\left(t_{w}-t_{*}\right)-t_{L}-t_{R}\right) .
\end{aligned}
$$




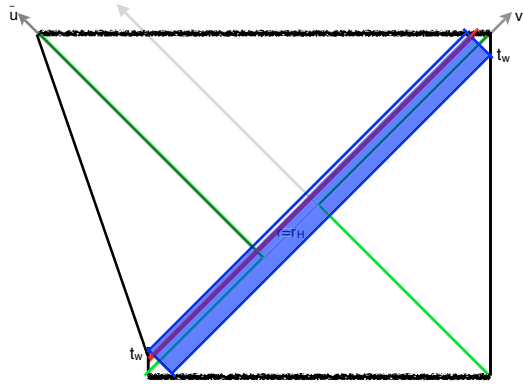

(a) Right after perturbation

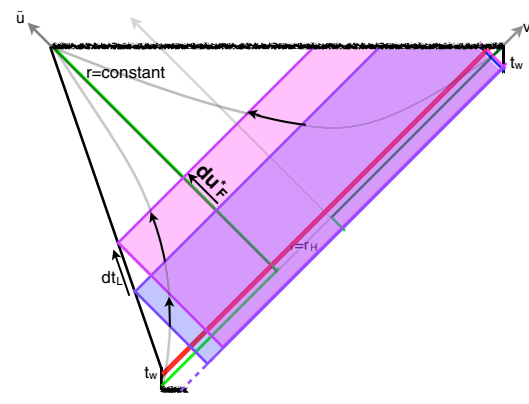

(b) $t_{L}$ goes up, $t_{R}$ fixed

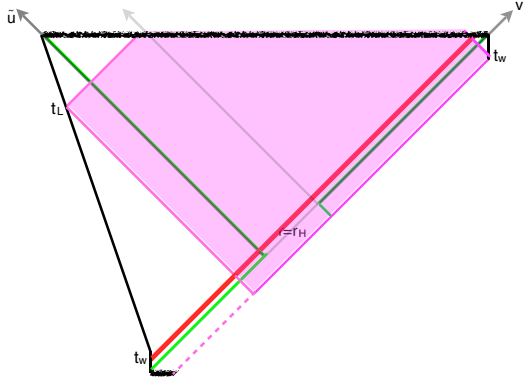

(c) $t_{w}-t_{L}>t_{*}$

FIG. 7. Evolution of the WDW patch as we increase the left time $t_{L}$.

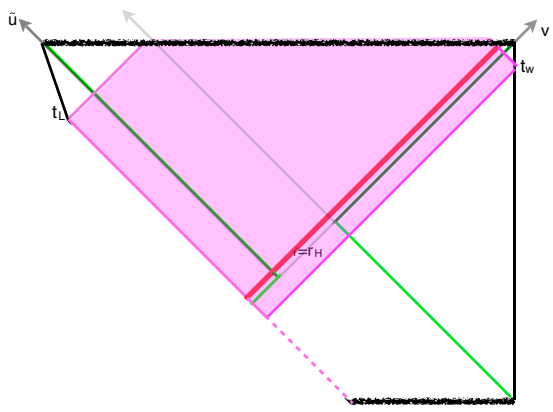

(a) $t_{R}=t_{w}$

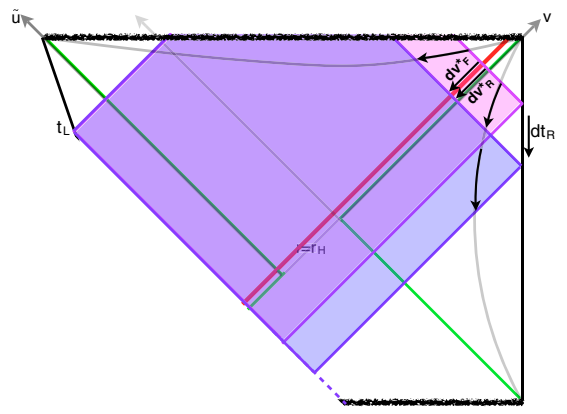

(b) $t_{R}$ goes down, $t_{L}$ fixed

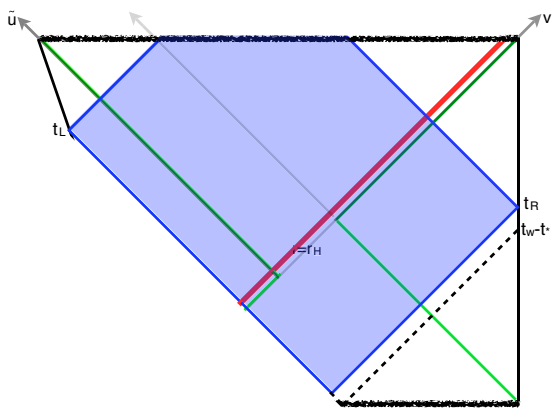

(c) $t_{w}-t_{R}<t_{*}$

FIG. 8. Evolution of WDW patch as we decrease the right time $t_{R}$.

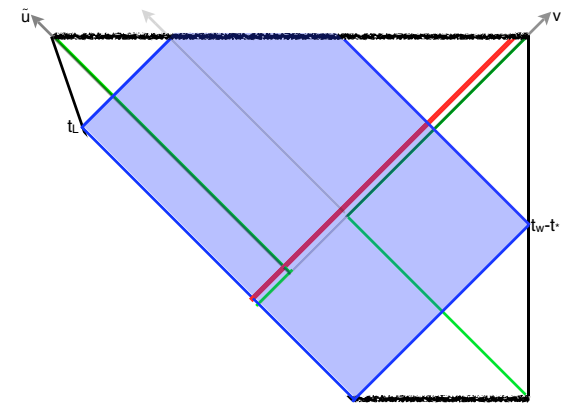

(a) $t_{R}=t_{w}-t_{*}$

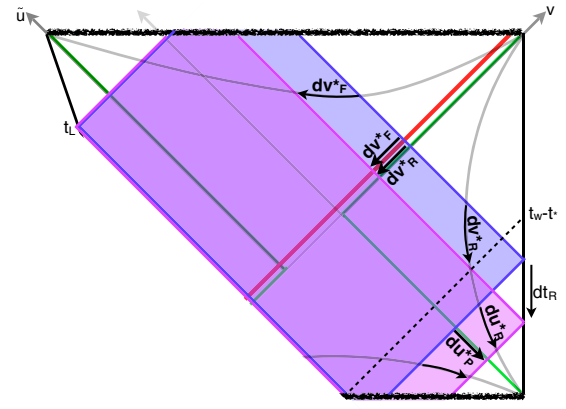

(b) $t_{R}$ goes down, $t_{L}$ fixed

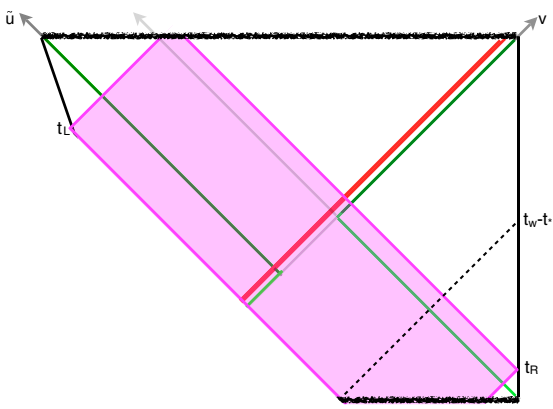

(c) $t_{w}-t_{R}>t_{*}$

FIG. 9. Evolution of WDW patch as we decrease the right time $t_{R}$.

Without perturbations, the answer would be $\mathcal{C}(|\mathrm{TFD}\rangle)+$ $C\left|t_{R}-t_{L}\right|$. We see that the time dependence becomes quite different in this regime. For example, let us set $t_{L}=-\infty$. $^{5}$ Without perturbations, the complexity will always increase with $t_{R}$. Now, with a shockwave present, the complexity will decrease with $t_{R}$ when $t_{w}-t_{R}>t_{*}$. This abnormal time dependence is a consequence of the breaking of boost symmetry as we go across the shockwave.

\footnotetext{
${ }^{5}$ This is just a technical choice to avoid a transient period. We will give more explanations about this in Sec. II D.
}

Combining Eqs. (2.9), (2.10), and (2.11), we recover the time dependence in Eq. (2.3).

In $D>3$, because the asymptotic boundaries bend outward, there will be a time of thermal length when the complexity is not changing. We do not understand the reason. In this paper, we shall consider it as part of the transient behavior and ignore it in our calculations.

In the above calculations, we ignored contributions from transient periods. It will depend on specific geometries and cannot be completely determined by a symmetry argument. However, in calculating the complexity of the precursor $W\left(t_{w}\right)$, the transient behavior has little effect. We carry out 
the calculation in Appendix A, and we see a smooth exponential-to-linear transition, as one expects from the analysis of quantum circuits.

The idea that boost symmetry determines the time dependence of complexity should be clear from the above calculations. Following the same procedure, we can consider more complicated examples. For states perturbed by multiple precursors, we follow the time evolution of preparing the state. Each time we make a perturbation, it takes a scrambling time for the memory of the previous perturbation to disappear, and the new perturbation takes a scrambling time to be squeezed up against the horizon. This is the origin of the $2 t_{*} n_{s b}$ subtractions in Eq. (2.4). The detailed calculations are in Appendix B.

For states with localized perturbations, the time dilation factors will be position dependent. From a symmetry point of view, not only is the boost symmetry broken, but the translation symmetry in the spatial transverse direction is also broken. But again, at a coarse-grained level, it is broken in a particularly simple pattern. More details are in Appendix C. When we vary the transverse positions of the scanning beam, ${ }^{6}$ we will also detect the transverse variations of geometry caused by the propagation of perturbations. Intuitively, one can imagine a tensor network lying at the stretched horizon. It evolves as the dynamics goes on. When we look at the complexity history, we scan this network. In particular, from the profile of complexity we can see that some part of the network is normal while some part is disrupted by high energy. This will be the topic of the next section: diagnosing the horizons by complexity.

\section{Time dependence of complexity as a diagnosis of good interior}

From earlier discussions, it should already be clear why we can use the complexity to detect the interiors of black holes or white holes. When there is nothing abnormal, the interior steadily expands as the boundary time evolves, and this will give a linear increase of complexity. An abnormal decrease of complexity means this evolution is disrupted.

There is one technical point here. There are four horizons in the example we discussed - both right and left future and past horizons. If we want to detect the transparency of one particular horizon, we do not want to be in a transient period; i.e., we want the boundary of the WDW patch to touch the singularity. To achieve this, we send the other time to infinity. For example, if we want to detect the transparency of the right future horizon, we set $t_{L}=-\infty$; i.e., we push the left time up to the upper corner. See Fig. 10. As we increase the right time, a linear increase in

\footnotetext{
${ }^{6}$ For localized perturbations, a scanning light beam is not a perfect analogy. With localized shockwaves, null geodesics do not stay at constant spatial positions. Nevertheless, we want to detect the interior along slices with constant spatial positions in order to compare with the case without perturbations.
}

complexity means that the part just scanned has a good interior [Fig. 10(a)]. If the complexity decreases somewhere, it means there is something wrong in that part of the interior [Fig. 10(b)].

If we want to detect the transparency of the right past horizon, we instead set the left time at the lower corner $\left(t_{L}=\infty\right)$ and scan the interior with Eddington-Finkelstein time $u^{*}=$ constant; see Fig. 11 .

Similarly, one can detect the smoothness of the left horizons by looking at the dependence of complexity on the left time. If we want to detect the upper-left horizon, we set $t_{R}=\infty$. If we want to look at the lower-left horizon, we set $t_{R}=-\infty$.

In the above examples of two-sided black holes, we anchored the time of the other side at infinity to avoid transient period. But for black holes with wide enough Penrose diagrams, this is unnecessary (Figs. 26 and 27). It is certainly unnecessary for one-sided black holes. In those cases, if we move time up, we are detecting the transparency of the upper horizon, while if we move time down, we are detecting the transparency of the lower horizon. Then a natural conjecture is, for a state which is not maximally complex and has both future and past horizons, at a fixed time, you cannot have firewalls on both of them. This is true in the examples we considered here. It also follows naturally from complexity considerations. The interiors store the quantum circuit making the state. Firewalls lock them up. But the complexity always changes before it reaches the maximum, so there must always be some horizon free of firewalls at work. More discussions on quantum circuits and firewalls are in Sec. III C.

To further study the connection between the time dependence of complexity and the smoothness of the horizon, we can look at the collision energy experienced by an infalling thermal quantum.

We assume there is a homogeneous perturbation, as in Sec. II C, in Fig. 5. Let us say that we throw in another thermal scale quantum from the right boundary at time $t_{R}$. It meets the matter shell at radius $r_{w}$. We have

$$
\frac{d v_{F}^{*}}{d v_{R}^{*}}\left(r_{w}\right)=\frac{r_{H}-r_{w}}{\delta r_{H}+r_{H}-r_{w}}=\frac{\left(r_{H}-r_{w}\right) / \delta r_{H}}{1+\left(r_{H}-r_{w}\right) / \delta r_{H}},
$$

where for a thermal scale quantum, $\delta r_{H} \sim \frac{r_{H}}{S} \sim \frac{l_{p}}{S^{(D-3) /(D-2)}}$, which is sub-Planck scale. On the other hand, the collision energy will be

$$
\begin{aligned}
\frac{E_{c}^{2}}{m_{p}^{2}} & =\frac{4 l_{p}^{2} E_{1} E_{2}}{f\left(r_{w}\right)} \sim \frac{l_{p}^{2} T}{r_{H}-r_{w}} \sim \frac{l_{p}^{2} T S}{r_{H}}\left[\left(\frac{d v_{F}^{*}}{d v_{R}^{*}}\right)^{-1}-1\right] \\
& \sim \frac{r_{H}}{\beta} S^{D-4}\left[\left(\frac{d v_{F}^{*}}{d v_{R}^{*}}\right)^{-1}-1\right] \propto\left(\frac{d v_{F}^{*}}{d v_{R}^{*}}\right)^{-1}-1 .
\end{aligned}
$$




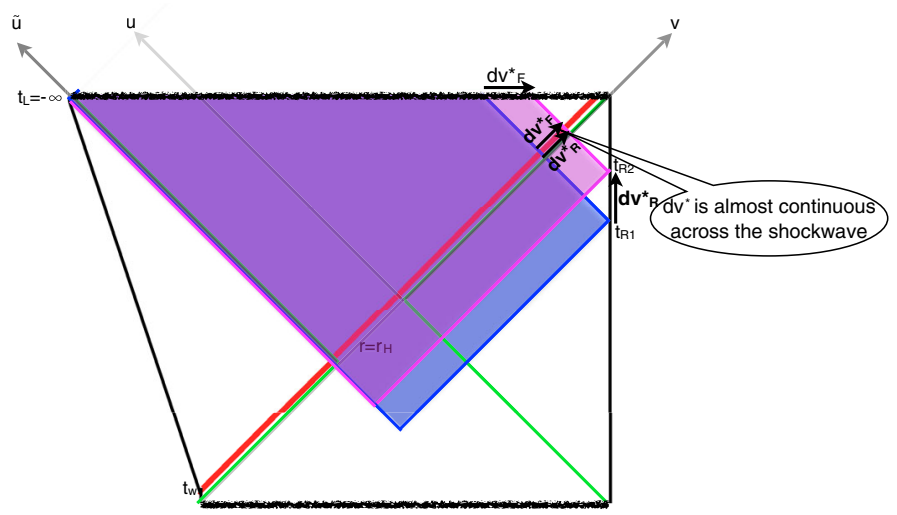

(a) When the energy of matter is mild, $d v^{*}$ is continuous and the complexity increases.

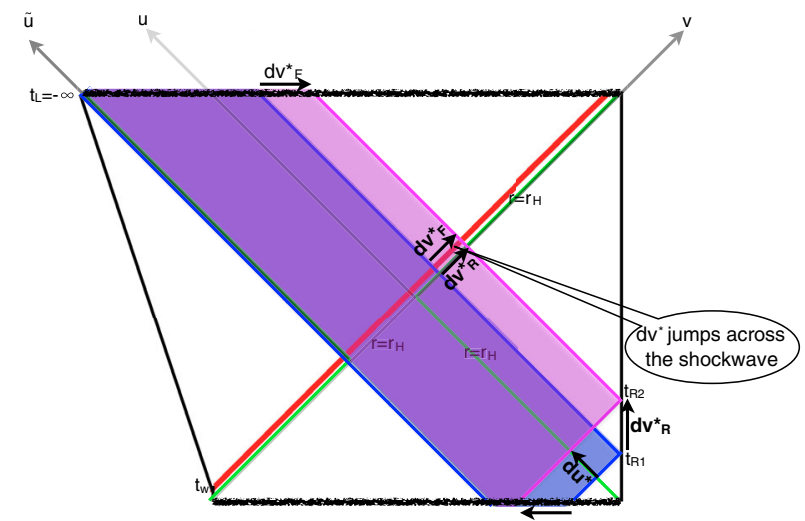

(b) When there is high energy matter, $d v^{*}$ jumps and the complexity decreases.

FIG. 10. To diagnose the smoothness of the right future horizon, we set $t_{L}$ at the top and scan the interior by setting the EinddingtonFinkelstein coordinates $v^{*}=$ constant.

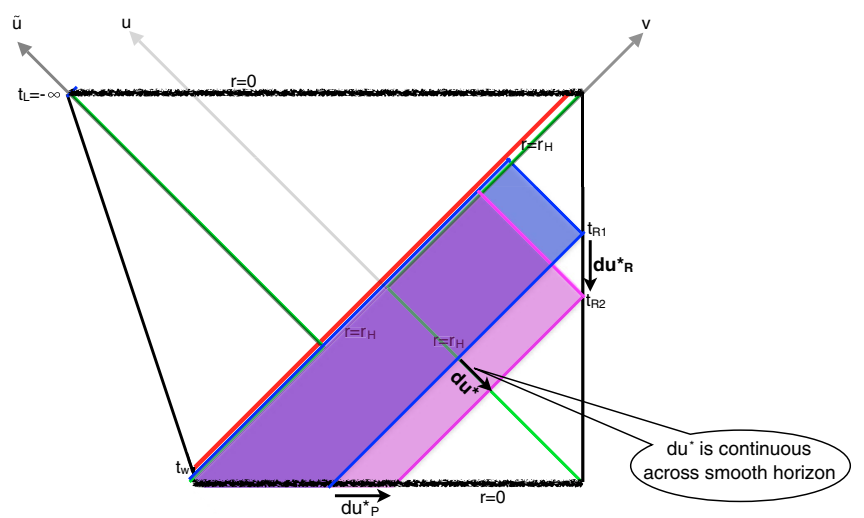

FIG. 11. To diagnose the smoothness of the right past horizon, we set $t_{L}$ at the bottom and scan the interior by setting the Eddington-Finkelstein coordinates $u^{*}=$ constant.

We see that with significant time dilation, $\frac{d v_{F}^{*}}{d v_{R}^{*}} \ll 1$. The collision energy squared is inversely proportional to the dilation factor and can reach the Planck scale.

More generally, in Sec. I of Appendix D we use the Raychaudhuri equation to relate the time dilation factors to the stress-energy tensors. Stress-energy tensors cause geodesics to focus. This fact can be translated into time dilation factors which control the time dependence of complexity.

There is one subtlety here. What do we mean by firewalls? Stress-energy tensors surely signal firewalls. They characterize energy from matter. But gravitons also carry energy. In fact, in the presence of localized perturbations, there can be nontrivial time dilation factors at places where the energy momentum tensor $T_{\mu \nu}$ vanishes. Someone who tries to cross the horizon there will still be hit hard, not by matter, but by gravitational shockwaves. We should also consider them as firewalls even though the local stressenergy tensors vanish. To quantitively describe this effect, we can use the Landau-Lifshitz pseudotensor [23].
The Landau-Lifshitz pseudotensor characterizes the energy-momentum flows carried by gravitons. It is only a tensor under linear coordinate transformations-in particular, Lorentz transformations. To describe the experience of an infalling observer, we should evaluate it in the local inertial frame right before the observer crosses the horizon. In Sec. II of Appendix D, we show that in the geometries we considered in this section, the collision energy is always controlled by the time dilation factors as in Eq. (2.12), whether it is from collisions with matter or with gravitons.

\section{QUANTUM CIRCUITS AND THE SMOOTHNESS OF HORIZONS}

\section{A. Future and past tapes}

Tensor networks support spacetime. As pointed out by Hartman and Maldacena [4], we can consider a tensor network laid near the horizon. As time grows, more layers are added to the tensor network, recording the action of the Hamiltonian. It was pointed out by Susskind that the minimal quantum circuit preparing a state is stored in a tape behind the future horizon (Fig. 12) [9]. It is called a tape because it keeps a record of the past actions of making the state. This picture is also the basic reason the

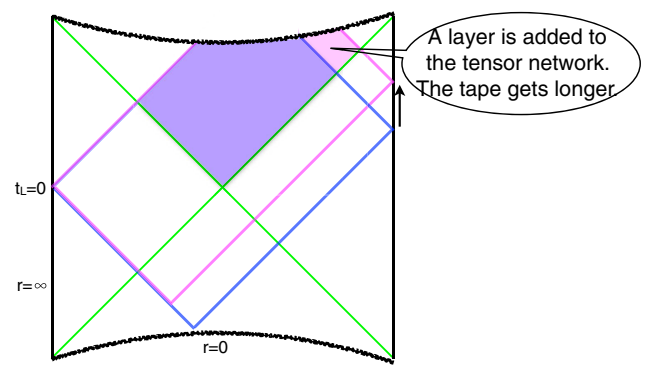

FIG. 12. The growth of the future tape. 


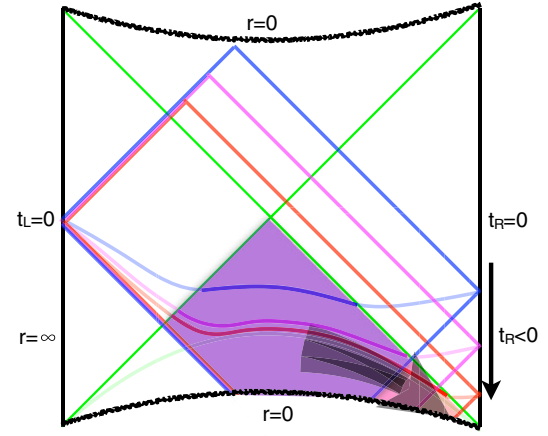

(a) The past tape

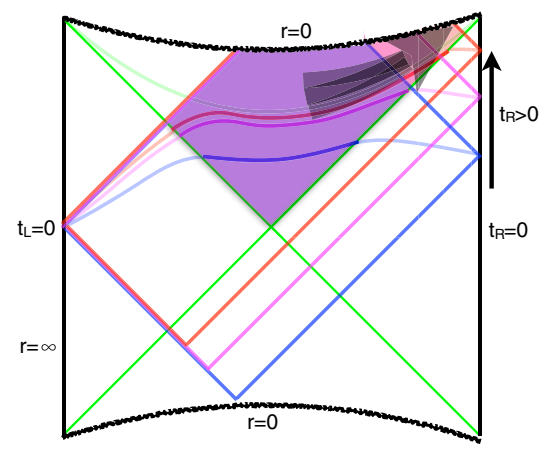

(b) The future tape

FIG. 13. The past and future tapes.

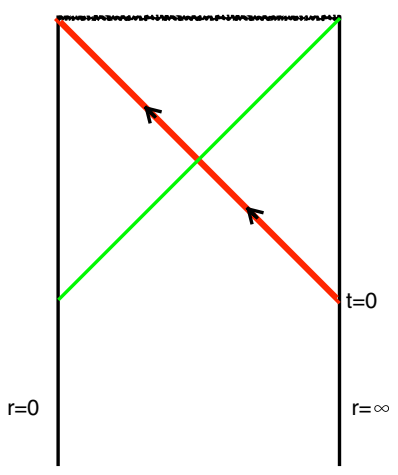

FIG. 14. A one-sided black hole formed by a collapsing shell.

complexity-volume duality works. For more details, see Sec. 3.3 and Figs. 10, 17, and 19 in Ref. [9].

In this section, we shall see that the future and past interiors are like two tapes storing different parts of the minimal circuit preparing the state. ${ }^{7}$ In the following sections, we will sometimes call the future interior the future tape, and the past interior the past tape.

Here is the simplest example: Consider the time evolution of a thermofield double. We fix $t_{L}=0$ and draw the WDW patches as well as the maximal surfaces at different $t_{R}$ 's (Fig. 13). When $t_{R}<0$, the past interior grows toward the past [Fig. 13(a)]. This is a white hole state, and the minimal circuit preparing this state is stored in the past tape. When $t_{R}>0$, the future interior expands toward the future [Fig. 13(b)]. This is a black hole state, and the minimal circuit is stored in the future tape.

Where are the tapes? There is only an AdS distance in the radial direction of the interior. When we talk about tapes, we mean this AdS-thickness thing (the colored regions in Fig. 13), which gets longer along the spacelike

\footnotetext{
${ }^{7}$ What we mean by future and past interiors should be clear from the context in the following discussions. Mathematically, we can consider the part of spacetime inside the future apparent horizon as the future interior, while the part inside the past apparent horizon is the past interior.
}

Schwarzschild time direction. We do not consider the tensor network to be on any specific slice. In complexity-volume duality $[5,7]$, the maximal-volume surface is used as a gauge-invariant way to represent the wormhole. As in Fig. 13, when the future or past interior expands, the maximal surface also goes through the future or past interior. But this does not mean that the particular slice is physically special. In fact, we cannot localize the tensor network to distances smaller than AdS scales.

\section{B. Two-tape picture: Why are there both future and past horizons?}

Let us make a more detailed comparison between the picture of the quantum circuit preparing a state and the dual black hole geometry. We shall see that the minimal circuit preparing a state can contain both forward and backward Hamiltonian evolutions [for example, the left and right legs in Fig. 15(b) for a precursor]. The forward Hamiltonian evolution part is stored in the future tape, while the backward Hamiltonian evolution part is stored in the past tape.

To avoid complications from the other side, in this section we will use a one-sided black hole as an example. We start from an AdS black hole formed from a collapsing shell at $t=0$ (Fig. 14).

Now, we perturb the state by inserting a precursor: $W\left(t_{w}\right)=e^{i H t_{w}} W e^{-i H t_{w}}$ with $t_{w}>t_{*}$. Figure 15(a) illustrates

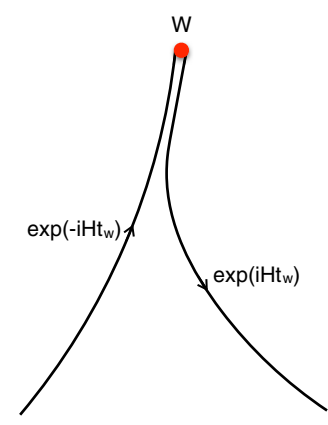

(a) One precursor

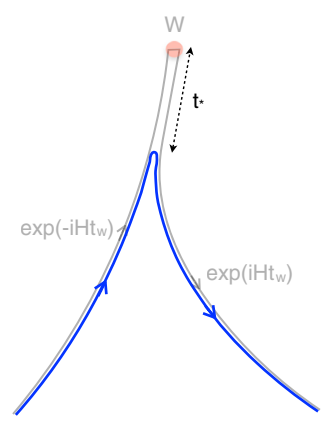

(b) Minimal circuit for one precursor
FIG. 15. One precursor. 


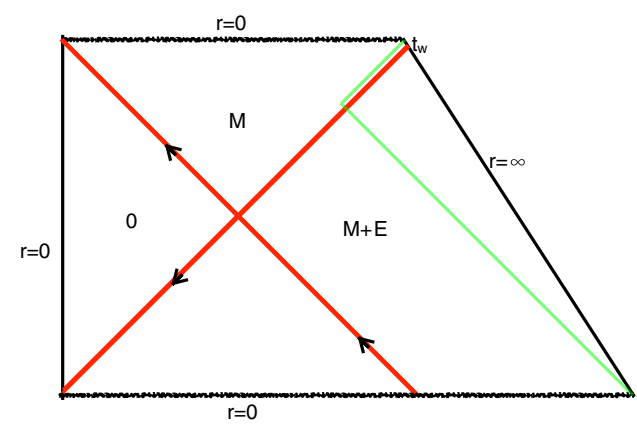

FIG. 16. A one-sided black hole perturbed by a precursor.

such a precursor composed of three parts: forward time evolution $e^{-i H t_{w}}$, perturbation $W$, and backward time evolution $e^{i H t_{w}}$. There will be cancellations right after the perturbation, and in Fig. 15(b) the blue line represents the minimal circuit after the cancellations. See Ref. [7] for more explanations.
With the perturbation, a past singularity will form, and the dual geometry looks like Fig. 16.

To match the quantum circuit picture with the black hole geometry, we start at $t=0$ when the black hole has just formed [Fig. 17(a)] and time-evolve it to $t=t_{w}$ [Fig. 17(b)]. The minimal circuit of this forward time evolution is shown as "A" in Fig. 17(c). It is stored in the future interior [" $A$ " in Fig. 17(b)]. In this regime, the white hole has not formed yet, and the future tape is working.

Next, we perturb the state by $W$ at $t=t_{w}>t_{*}$, and then evolve it backward. We start right after the perturbation at $t=t_{w}$ [Fig. 18(a)], where the minimal circuit "A" [Fig. 18(b)] is stored in the future interior ["A" in Fig. 18(a)]. A white hole forms.

We evolve backward, until $t=t_{w}-t_{*}$. When $t_{w}-t<t_{*}$, the backward time evolution will cancel part of the earlier forward time evolution [In Fig. 19(b), the circuit labeled "B" cancels part of the circuit "A," resulting in the minimal circuit " $\mathrm{C}$ "] [7]. As a result, the minimal

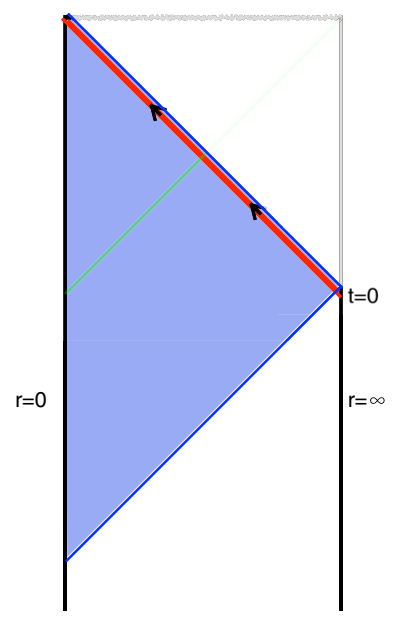

(a)

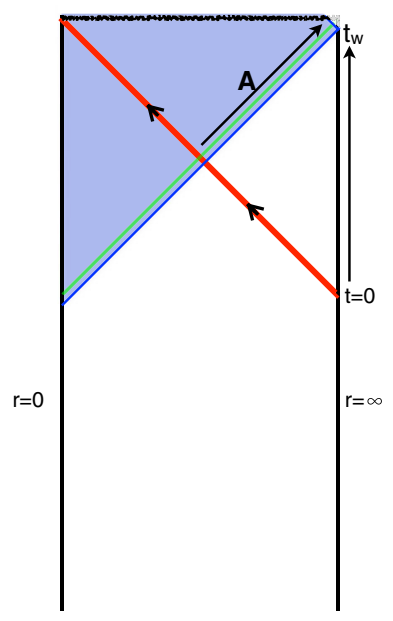

(b)

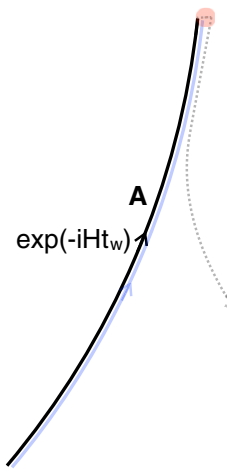

(c)

FIG. 17. A black hole forms.

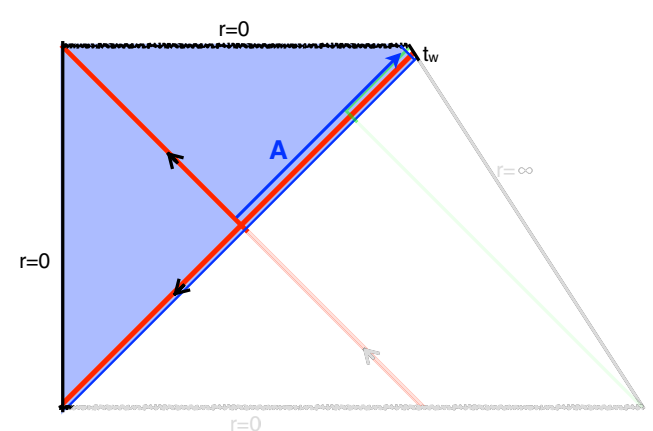

(a) Bulk geometry right after the perturbation

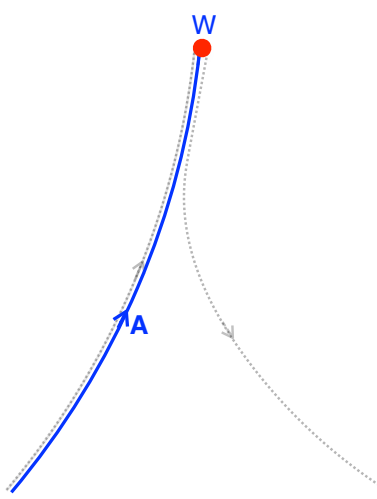

(b) Quantum circuit right after the perturbation

FIG. 18. A white hole forms. 


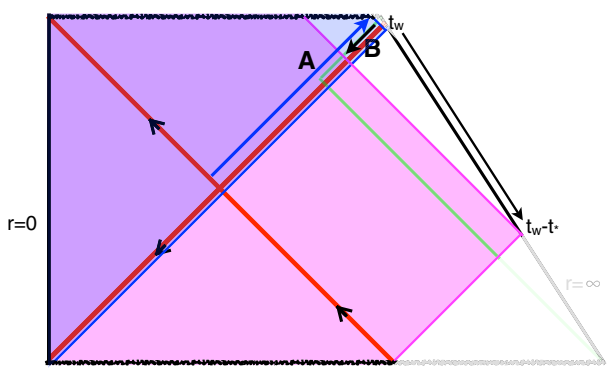

(a)

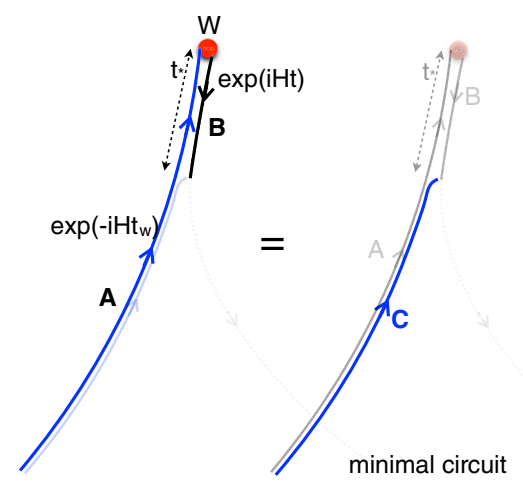

(b)

FIG. 19. Making the state by forward Hamiltonian evolution.

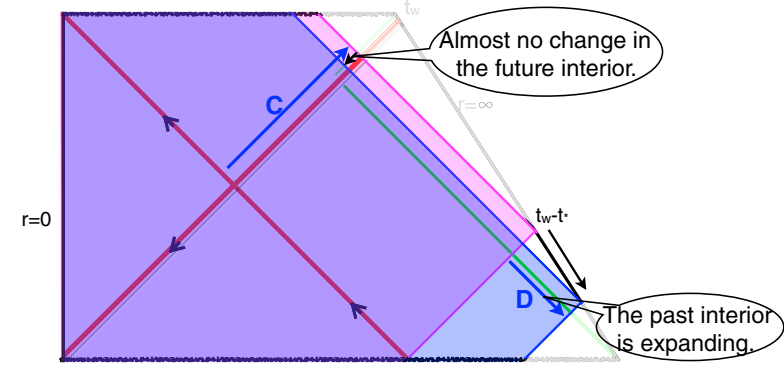

(a)

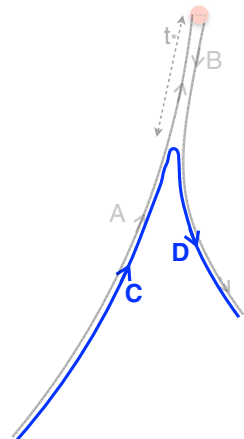

(b)

FIG. 20. Making the state by backward Hamiltonian evolution.

circuit gets smaller, and it still only contains forward Hamiltonian evolution ["C" in Fig. 19(b)]. On the bulk side, the future interior shrinks [Fig. 19(a)]. We ignore the change in the past interior, since the volume inside the postcollision region is small. So we see that the minimal circuit making the state at this time $\left(t=t_{w}-t_{*}\right)$, which contains only forward Hamiltonian evolution, is still stored in the future interior.

Next, we further decrease time $t$. By now $\left(t<t_{w}-t_{*}\right)$, the perturbation has spread to the entire system, and there are no more cancellations between the backward and forward Hamiltonian evolutions. The minimal circuit is growing from backward Hamiltonian evolution [" $D$ " in Fig. 20(b)]. On the bulk side, the future interior does not change, because there is significant time dilation across the firewall [Fig. 20(a)]. The past interior is expanding, and the circuit from backward Hamiltonian evolution is stored there ["D" in Fig. 20(a)].

Eventually, we get to $t=0$, and we have made the state perturbed by the precursor $W\left(t_{w}\right)$. In the minimal circuit making this state, the forward Hamiltonian evolution ["C" $\mathrm{C}$ " in Fig. 21(b)] is stored in the future interior ["C" in Fig. 21(a)], while the backward Hamiltonian evolution ["D" in Fig. 21(b)] is stored in the past interior ["D" in Fig. 21(a)].
We see the roles played by future and past horizons. While the stretching of the future interior is responsible for the increase of complexity to the future, to have complexity decrease, it seems necessary to have past horizons. Complexity is stored in interiors. The existence of past horizons means the complexity can also increase towards the past, and so it is a manifestation of time reversibility.

\section{Tape working and the smoothness of horizons}

There is one confusing point about the relation between the time dependence of complexity and the smoothness of horizons. The time dependence of complexity is not a conventional property of states in general. There are properties of states described by linear operators, like position, momentum, etc. Entanglement is also a property of states, though it may not always be linear [24]. These properties are independent of dynamics. But given a state, without knowing the Hamiltonian, in general one cannot talk about the time dependence of its complexity. If the smoothness of a horizon is a state property, then how is it related to the time dependence of complexity? The twotape picture gives an answer to this question. 


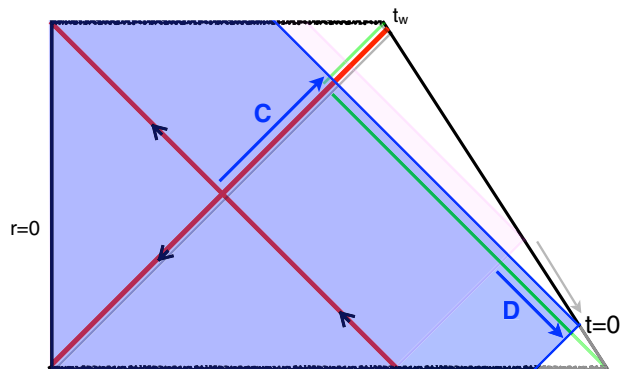

(a)

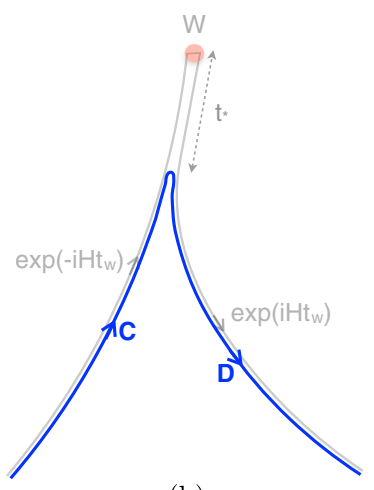

(b)

FIG. 21. The future and past interiors store different parts of the circuits.

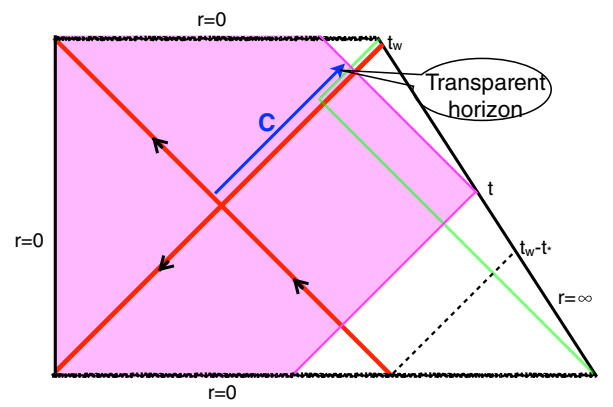

(a)

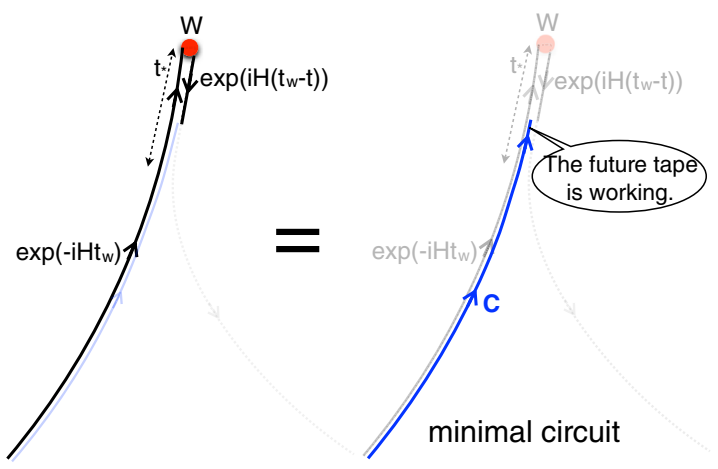

(b)

FIG. 22. Future tape working.

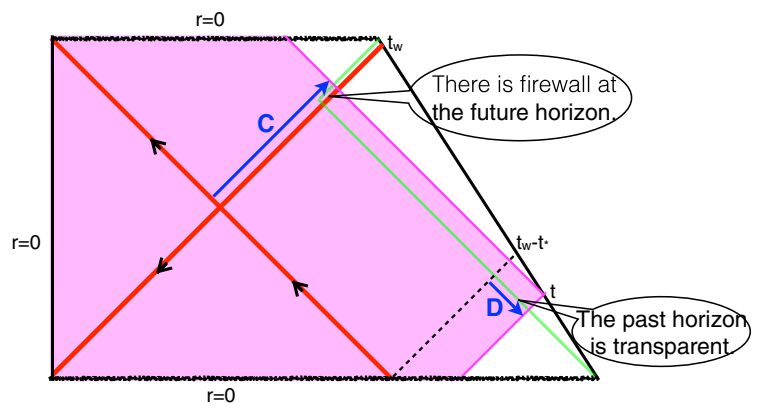

(a)

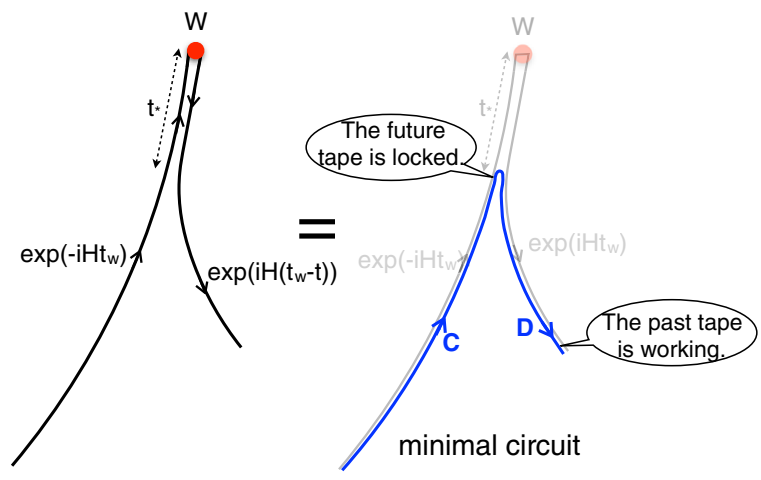

(b)

FIG. 23. Past tape working.

Let us look at the perturbed one-sided black hole at a particular time $t>t_{w}-t_{*}$ [Fig. 22(a)]. We have seen that its complexity increases with time. The perturbation is not too close to the horizon.

The minimal circuit preparing this state is shown as the blue line " $\mathrm{C}$ " in Fig. 22(b). At the end of the minimal circuit, it is forward Hamiltonian evolution. The quantum gates making the state are being laid on the future tape, so we say the future tape is working. This is why the complexity will increase as we increase the time. Because the tensor network supports spacetime, this also implies that the future interior is growing. The future horizon is transparent here. The past tape is not working, and inside the past interior there is a highenergy collision. 
If we look at the state at another time $t<t_{w}-t_{*}$, the minimal circuit will look completely different. Now the minimal circuit [the blue line in Fig. 23(b)] contains both forward Hamiltonian evolution ["C" in Fig. 23(b)] and backward Hamiltonian evolution ["D" in Fig. 23(b)]. The forward-evolution part is stored in the future tape ["C" in Fig. 23(a)], while the backward-evolution part is stored in the past tape ["D" in Fig. 23(a)]. At the end of the minimal circuit, it is backward Hamiltonian evolution; i.e., it is the past tape working. No quantum gates are being laid on the future tape at this moment, and we say that the future tape is locked. From the complexity point of view, we see the complexity decrease because the past tape working means the complexity is increasing towards the past.

We see that the smoothness of horizons has a simple interpretation in this picture. The future and past interiors are like two tapes storing different parts of the minimal circuit. Each tape can be in two states: working, when there are quantum gates being laid on it, or locked, when no more gates are being laid on it. When a tape is working, it is getting longer from those newly added quantum gates. So the corresponding interior steadily grows with time. At this moment, the interior is open for someone to enter. When a tape is locked, no change happens at the end of it. As a result, the corresponding interior ceases to grow. Nothing can fall in at this moment.

\section{CONCLUSION}

We showed that the time dependence of complexity is closely related to the boost symmetry across horizons. The Rindler-like nature of horizons guarantees that the boost symmetry is broken in simple and universal forms, which gives rise to the various robust features of the time dependence of complexity.

By identifying the black and white hole interiors as tapes storing different parts of the minimal circuit preparing the state, we see the various ideas fit together. The tape working means the interior growing, the boost symmetry staying good, and a transparent horizon. On the other hand, a tape being locked means the interior stops growing, the boost symmetry is broken, and any infalling object will experience a high-energy collision.

It would be interesting to understand this boost symmetry and symmetry breaking in the boundary field theory. Intuitively, without perturbations, there is uniform dynamics. Right after the perturbation, the perturbation is distinguishable, and it is not very symmetric. After it is scrambled, locally everywhere is the same, and a certain symmetry is regained. A steady increase of complexity is a kind of time translation symmetry.

In our analysis, we ignored various regions of AdS size and considered them as giving rise to transient behaviors. However, they serve as the "seeds" for later steady expansion of the interior. On the quantum circuit side, they correspond to the most interesting behavior of switching from one tape to another. But without an understanding of sub-AdS locality, we do not have tools to address them.

Another problem is about the linearity of firewalls. If we want to see whether the superposition of two states has a transparent horizon, we need to know something about the minimal circuit preparing the superposition. Unfortunately, so far we know very little about the complexity of superpositions without using ancilla qubits. ${ }^{8}$ Maybe a more basic question to ask is "On what states can we talk about superpositions?" It may not make sense to talk about superpositions of states with large relative complexity. One possibility is that the existence of firewalls behaves like an ordinary property only if we restrict to a certain subspace of states, like entanglement entropy as shown by Harlow recently in Ref. [24]. Another possibility is that to describe black hole interiors we indeed need some violations of quantum mechanics, but no observer should be able to see it. After all, testing if there exists a firewall or not is not some experiment that can be repeated by a single observer.

A key assumption we used throughout this paper is the validity of classical general relativity, at least until very late times. This assumption might break down earlier. But it is interesting to see how far we can go with these assumptions. We see that all these ideas-complexity, minimal circuit, and firewalls-fit together, at least in the examples we studied. However, we do keep in mind that these examples can be special. Furthermore, it may be a very strong constraint to require that a state determine the minimal circuit making it. To further address this, a better understanding of complexity and minimal circuits is needed.

\section{ACKNOWLEDGMENTS}

I am grateful to my advisor Lenny Susskind for tremendous help in various aspects. I thank Scott Aaronson for explanations on the complexity of superposition. I acknowledge support from the Simons Foundation through the It from Qubit collaboration and from Stanford Institute for Theoretical Physics.

\section{APPENDIX A: COMPLEXITY OF A PRECURSOR}

Our goal is to calculate the complexity of the precursor $W\left(t_{w}\right)=e^{i H t_{w}} W e^{-i H t_{w}}$. Consider the complexity of $W_{L}\left(t_{w}\right)$ $|\mathrm{TFD}\rangle$. Geometrically, we start from $t_{R}=t_{L}=t_{w}$ [Fig. 24(a)], decrease $t_{R}=t_{L}=t$ [Fig. 24(b)] until $t=0$ [Fig. 24(c)]. During this process, the tip of the WDW patch never leaves the singularity, so the symmetry argument always works.

\footnotetext{
${ }^{8}$ We thank Scott Aaronson for explanations on this.
} 


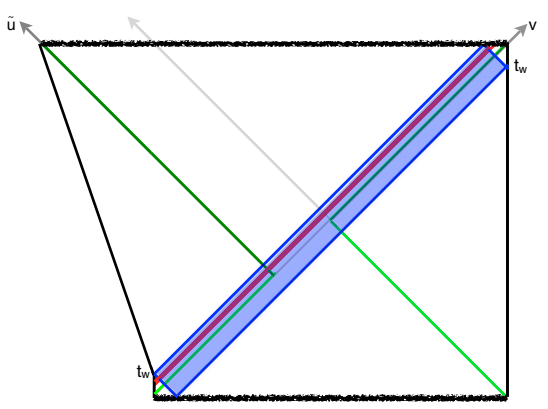

(a) $t_{R}=t_{L}=t_{w}$

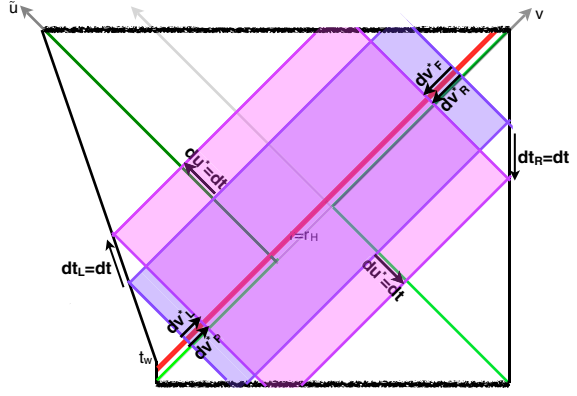

(b) Change both $t_{R}$ and $t_{L}$ by $d t$.

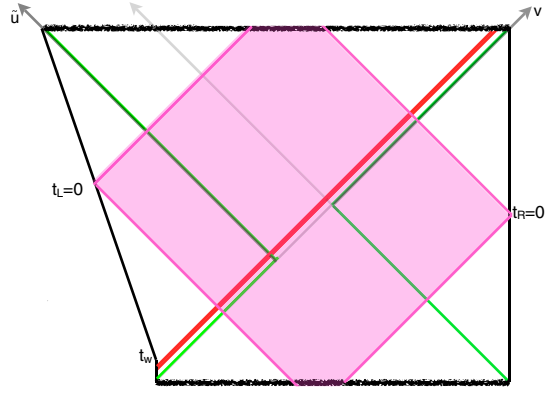

(c) $t_{R}=t_{L}=0$

FIG. 24. Complexity of a precursor.

We have

$$
\begin{aligned}
\frac{d \mathcal{C}_{1}(t, t)}{d t} & =C\left(\frac{d v_{F}^{*}}{d v_{R}^{*}}-1+\frac{d v_{P}^{*}}{d v_{L}^{*}}-1\right)=-2 C \frac{c e^{\frac{2 \pi}{\beta}\left(t_{w}-t_{*}-t\right)}}{1+c e^{\frac{2 \pi}{\beta}\left(t_{w}-t_{*}-t\right)}}, \\
\mathcal{C}_{1}(t, t) & =\mathcal{C}(|\mathrm{TFD}\rangle)+2 C \frac{\beta}{2 \pi} \log \left(1+c e^{\frac{2 \pi}{\beta}\left(t_{w}-t_{*}-t\right)}\right) .
\end{aligned}
$$

With $t=0$, we get the complexity of a precursor:

$$
\mathcal{C}\left(W\left(t_{w}\right)\right)=2 C \frac{\beta}{2 \pi} \log \left(1+c e^{\frac{2 \pi}{\beta}\left(t_{w}-t_{*}\right)}\right) .
$$

This has exactly the same functional form as the geodesic distance in the BTZ black hole [12]. The symmetry argument will be accurate when $t_{w}$ is a fraction of scrambling time (when the Rindler approximation is good). Since this calculation only depends on the boost symmetry and the Rindler nature of horizons, it must reflect some universal property of horizon dynamics. It is interesting to see the exact functional form also come out of a simple epidemic model [17].

\section{APPENDIX B: SWITCHBACK FROM MULTIPLE PRECURSORS}

In Sec. II C, we studied in detail the case of one precursor, and we saw that the time dependence of complexity is determined by the behavior of boost symmetry across the horizon. Let us see how this works with multiple perturbations separated by large times: $W\left(t_{w 2}\right) W\left(t_{w 1}\right)|\mathrm{TFD}\rangle$. We assume $t_{w 1}>t_{*}$, and $t_{w 2}<-t_{*}$. See Fig. 25 [13].

At $t_{L}=t_{w 2}$, the effect of the second perturbation is small, and we essentially have a state with one shockwave [Fig. 26(a)]. We have its complexity from a previous section [Eqs. (2.9), (2.10), and (2.11)].

Now we evolve $t_{L}$ downward. The black hole interior shrinks, but inside the white hole, we will encounter a postcollision region [Fig. 26(b)]. The collision energy

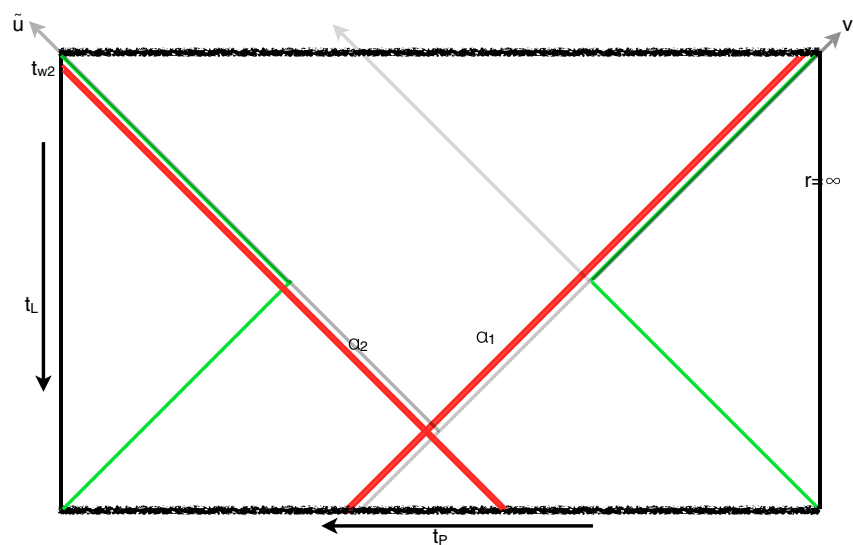

FIG. 25. Thermofield double perturbed by two precursors separated by a large time.

increases exponentially with $t_{w 1}-t_{w 2}$. With $t_{w 1}-t_{w 2}>2 t_{*}$, it will be comparable to the mass of the black hole, but the size of the spacetime region there is exponentially small. Here, we use assumption 4 to ignore its contribution. ${ }^{9}$ It takes the scrambling time for the first shockwave to fall into the singularity. In this regime $\left(t_{L}-t_{w 2}<t_{*}\right)$,

$$
\begin{aligned}
\frac{d \mathcal{C}_{2}\left(t_{L}, t_{R}\right)}{d t_{L}} & =-C \frac{d u_{F}^{*}}{d u_{L}^{*}} \\
\mathcal{C}_{2}\left(t_{L}, t_{R}\right) & =\mathcal{C}_{1}\left(t_{L}=t_{w 2}, t_{R}\right)-C\left(t_{L}-t_{w 2}\right) .
\end{aligned}
$$

Notice that during this regime, when $0<t_{L}-t_{w 2}<t_{*}$ as we evolve $t_{L}$ down, the white hole interior does not expand much due to the collision, and the black hole interior shrinks because the new perturbation is still mild. This will give rise to $2 t_{*}$ switchback. If we ever want to compare black hole geometry with the quantum circuit picture, this regime corresponds to the cancellations between forward and backward time evolutions.

\footnotetext{
${ }^{9}$ We are aware that the high energy here could lead to complications. The circuit picture indicates that there are no significant additional contributions.
} 


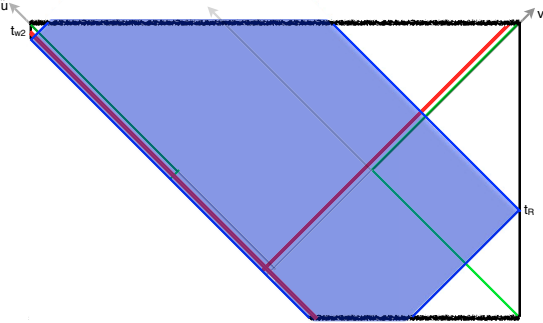

(a) Right after the second perturbation

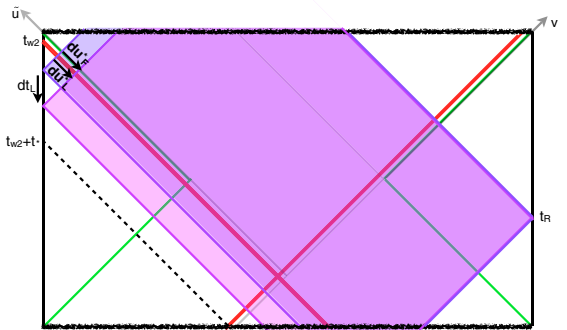

(b) Decrease $t_{L}$

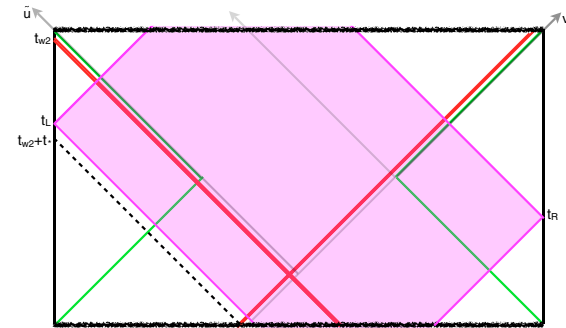

(c) $t_{L}-t_{w 2}<t_{*}$

FIG. 26. Less than the scrambling time after the second perturbation.

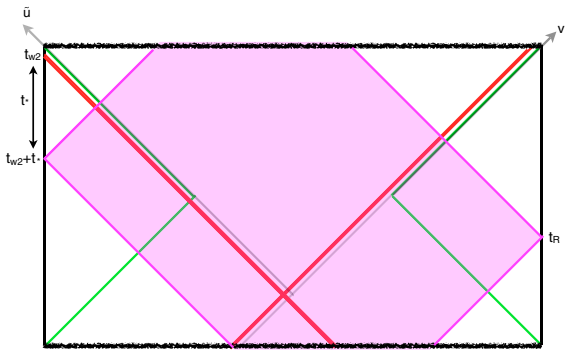

(a) Scrambling time after the second perturbation

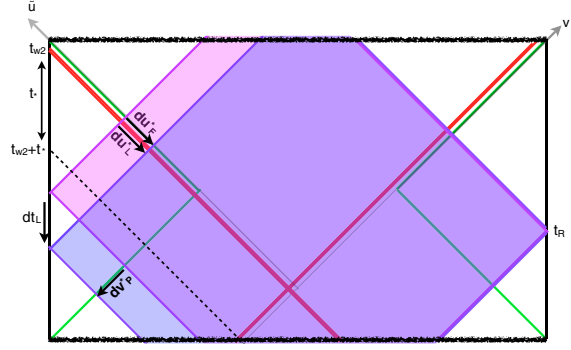

(b) Further decrease $t_{L}$

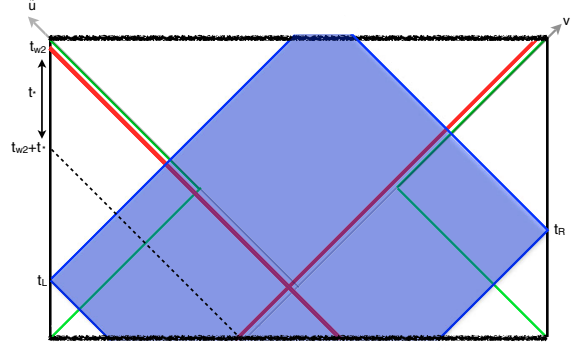

(c) $t_{L}-t_{w 2}>t_{*}$

FIG. 27. More than the scrambling time after the second perturbation.

As we further push $t_{L}$ down, once $t_{L}-t_{w 2}>t_{*}$, we leave the collision region (Fig. 27). The white hole starts to expand, while the shrinking of the black hole is significantly delayed due to the second shockwave.

We have

$$
\begin{aligned}
\frac{d \mathcal{C}_{2}\left(t_{L}, t_{R}\right)}{d t_{L}}= & C\left(1-\frac{d u_{F}^{*}}{d u_{L}^{*}}\right)=C \frac{c e^{\frac{2 \pi}{\beta}\left(t_{L}-t_{w 2}-t_{*}\right)}}{1+c e^{\frac{2 \pi}{\beta}\left(t_{L}-t_{w 2}-t_{*}\right)}} \\
\mathcal{C}_{2}\left(t_{L}, t_{R}\right)= & \mathcal{C}_{2}\left(t_{w 2}+t_{*}, t_{R}\right)+C \frac{\beta}{2 \pi} \log \left(1+c e^{\frac{2 \pi}{\beta}\left(t_{L}-t_{w 2}-t_{*}\right)}\right) \\
& -C \frac{\beta}{2 \pi} \log \left(1+c e^{\frac{2 \pi}{\beta}\left(t_{w 2}+t_{*}-t_{w 2}-t_{*}\right)}\right) \\
= & \mathcal{C}_{1}\left(t_{L}=t_{w 2}, t_{R}\right)+C\left(t_{L}-t_{w 2}-2 t_{*}\right)
\end{aligned}
$$

We notice that in this regime, as we push $t_{L}$ up, the complexity will decrease, which reflects the fact that the left future horizon is not transparent.

Now, we look at the regime when $t_{L}-t_{w 2}>t_{*}$, $t_{w 1}-t_{R}>t_{*}$. Plugging in $\mathcal{C}_{1}\left(t_{L}=t_{w 2}, t_{R}\right)$ from Eq. (2.11), we have

$$
\begin{aligned}
\mathcal{C}_{2}\left(t_{L}, t_{R}\right)= & \mathcal{C}(|\mathrm{TFD}\rangle)+C\left[2\left(t_{w 1}-t_{*}\right)-t_{R}\right. \\
& \left.+2\left(-t_{w 2}-t_{*}\right)+t_{L}\right] .
\end{aligned}
$$

There are two switchbacks, exactly as in Eq. (2.4).

\section{APPENDIX C: BREAKING SPATIAL TRANSLATION SYMMETRY: LOCALIZED SHOCKS}

We can also look at localized perturbations. Their holographic duals are black holes with localized shocks [5]. Assume a planar black hole without perturbations:

$$
d s^{2}=l^{2}\left(-f(r) d t^{2}+\frac{d r^{2}}{f(r)}+r^{2} d x^{i} d x^{i}\right),
$$

where $f(r)=r^{2}-r^{3-D}$.

With localized perturbation at an early time, $W_{x_{w}}\left(t_{w}\right)=$ $e^{i H t_{w}} W_{x_{w}} e^{-i H t_{w}}$, null lines of constant $v^{*}$ and $x$ again will be pushed forward, ${ }^{10}$ but this time in an $x$-dependent way. It was shown in Ref. [5] that at large $\left|x-x_{w}\right|$, when crossing the horizon, the null lines will be pushed forward in Kruskal coordinates by the amount

$$
\begin{aligned}
h(x) & =\frac{\exp \left[\frac{2 \pi}{\beta}\left(t_{w}-\frac{\left|x-x_{w}\right|}{v_{B}}-t_{*}\right)\right]}{\left|x-x_{w}\right|^{\frac{D-3}{2}}} \\
& =\exp \left[\frac{2 \pi}{\beta}\left(t_{w}-\frac{\left|x-x_{w}\right|}{v_{B}}-t_{*}\right)-\frac{D-3}{2} \log \left|x-x_{w}\right|\right],
\end{aligned}
$$

where $v_{B}=\sqrt{\frac{(D-1)}{2(D-2)}}$.

\footnotetext{
${ }^{10}$ Contrary to the case with homogeneous perturbations, they are no longer null geodesics.
} 
This again will cause a mismatch between EddingtonFinkelstein time inside and outside the horizon, in an $x$-dependent way. If we look at Eq. (C1), when $\left|x-x_{w}\right| \gg 1$, inside the exponential the linear term dominates over the logarithmic term, and the perturbation to spatial location $x$ effectively starts at time $t_{w}-\frac{\left|x-x_{w}\right|}{v_{B}}$, so there will be $x$-dependent time delay. If we look at a fixed value $x$, the situation is similar to the homogeneous case as discussed in earlier sections once we replace $t_{w}$ with $t_{w}-\frac{\left|x-x_{w}\right|}{v_{B}}$

Now, let us consider the complexity of this perturbed state. We can first consider the contribution from a fixed $x$ slice, which we know the appearance of from earlier discussions, and then perform an integration in $x$. Again, when we do this, we implicitly use assumption 2 ; i.e., the locality of the tensor network down to AdS scale. Here, a fixed $x$ slice has width $l_{\text {AdS }}$. So far, we have recovered all time dependence of complexity, as discussed in Sec. II A.

\section{APPENDIX D: COLLISION ENERGY AND TIME DILATION FACTORS}

\section{Raychaudhuri equation}

We have seen that we can use a bundle of ingoing light rays to detect the change of Eddington-Finkelstein time across the horizon, and hence detect the time dependence of complexity. The Raychaudhuri equation describes the behaviors of a congruence of geodesics [25]—in particular, how they respond to stress-energy tensors.

Consider a bundle of ingoing light rays. This bundle can either expand or shrink as they travel. To quantitatively describe this, consider a small area element $A$ in transverse directions. The expansion rate $\theta$ is defined as

$$
\frac{d A}{d \tau}=\theta A
$$

The Raychaudhuri equation gives

$$
\frac{d \theta}{d \tau}=-R_{a b} E_{0}^{a} E_{0}^{b}-2 \sigma^{2}+2 \omega^{2}-\frac{1}{D-2} \theta^{2}
$$

where $\omega$ reflects the rotation of neighboring light rays, $\sigma$ reflects the distortion of shapes, and $\theta$ reflects the area expansion. $E_{0}=\partial_{\tau}$ is the vector field along proper time. From the term $R_{a b} E_{0}^{a} E_{0}^{b}=8 \pi G_{N} T_{a b} E_{0}^{a} E_{0}^{b}$, we see that the presence of energy causes the bundle of light rays to focus.

With a shell of matter present, the expansion rate $\theta$ can suddenly jump, while there will be time dilation $\frac{d v^{*}}{d v^{*}}$ across the shell. In the case of homogeneous perturbations, radial light rays stay on constant transverse positions as they travel. We have $\frac{d \tilde{v}^{*}}{d v^{*}}=\frac{\theta}{\tilde{\theta}}=\frac{\theta}{\theta+\Delta \theta}, \quad$ i.e., $\quad \frac{\Delta \theta}{\theta}=\left(\frac{d \tilde{v}^{*}}{d v^{*}}\right)^{-1}-1$.

With localized perturbations, light rays will be refracted, and the time dilation factors will depend on transverse positions. This will give additional contributions to the expansion rate:

$\frac{\Delta \theta}{\theta}=\left[\left(\frac{d \tilde{v}^{*}}{d v^{*}}\right)^{-1}-1\right]-\left(\frac{\beta v_{B}}{2 \pi}\right)^{2} \nabla^{2}\left[\left(\frac{d \tilde{v}^{*}}{d v^{*}}\right)^{-1}-1\right]$,

where the Laplacian in Eq. (D4) is in transverse $x^{i}$ directions. ${ }^{11}$

On the other hand, from Eqs. (D1) and (D2), we have

$$
\begin{aligned}
\frac{d \theta}{\theta}= & -\frac{1}{D-2} \frac{d A}{A} \\
& \times\left[1+(D-2)\left(\frac{8 \pi G_{N} T_{a b} E_{0}^{a} E_{0}^{b}+2 \sigma^{2}-2 \omega^{2}}{\theta^{2}}\right)\right] .
\end{aligned}
$$

In the limit of a thin shell, $\frac{\Delta A}{A} \rightarrow 0$, and there will not be singularities in $\omega$ and $\sigma$, since they reflect rotations and separations of neighboring light lays. So the only potential contribution to the sudden jump of the expansion rate comes from the stress-energy tensor term $\frac{\Delta A}{A} \frac{8 \pi G_{N} T_{a b} E_{0}^{a} E_{0}^{b}}{\theta^{2}}$. This term is proportional to the collision energy squared. Near horizons, the large relative boost between the matter and the detecting light beam makes it grow exponentially in time. Combining this with Eq. (D3), we see that with homogeneous perturbations, the collision energy squared with matter is inversely proportional to the time dilation factor.

With localized perturbations [5], $T_{a b}$ has localized support in transverse spatial directions: $T \propto E e^{\frac{2 \pi}{\beta} t_{w}} \delta^{D-2}$ $\left(x-x_{w}\right)$. The solution of Eq. (D4) shows that the time dilation factors $\frac{d \tilde{v}^{*}}{d v^{*}}$ can still be proportional to $E e^{\frac{2 \pi}{\beta} t_{w}}$, even at positions where $x \neq x_{w}$ and $T$ vanishes. Someone falling in there will not encounter matter, but will still be hit hard by gravitational shockwaves. In the next section, we will estimate the collision energy by evaluating the LandauLifshitz pseudotensors.

This shows how the presence of the stress-energy tensor affects boost symmetry across horizons. Equations (D3)

\footnotetext{
${ }^{11}$ Equations (D3) and (D4) are exact in the limit of a thin matter shell on the horizon. Away from the horizon, $T_{a b} E_{0}^{a} E_{0}^{b}$ will not get too large, and matter will not cause a significant jump in the expansion rate.
} 
and (D4) relate the change of expansion rate to EddingtonFinkelstein time dilation, which controls the time dependence of complexity, while the Raychaudhuri equation [Eq. (D5)] shows that a sudden change of expansion rate is related to high collision energy.

\section{Landau-Lifshitz pseudotensor}

To see the energy carried by gravitons, we look at the Landau-Lifshitz pseudotensor [23]:

$$
\begin{aligned}
t^{\mu \nu}= & \frac{1}{16 \pi G_{N}}\left[\left(g^{\mu a} g^{\nu b}-g^{\mu \nu} g^{a b}\right)\left(2 \Gamma_{a b}^{c} \Gamma_{c d}^{d}-\Gamma_{a d}^{c} \Gamma_{b c}^{d}-\Gamma_{a c}^{c} \Gamma_{b d}^{d}\right)\right. \\
& +g^{\mu a} g^{b c}\left(\Gamma_{a d}^{\nu} \Gamma_{b c}^{d}+\Gamma_{b c}^{\nu} \Gamma_{a d}^{d}-\Gamma_{c d}^{\nu} \Gamma_{a b}^{d}-\Gamma_{a b}^{\nu} \Gamma_{c d}^{d}\right) \\
& +g^{\nu a} g^{b c}\left(\Gamma_{a d}^{\mu} \Gamma_{b c}^{d}+\Gamma_{b c}^{\mu} \Gamma_{a d}^{d}-\Gamma_{c d}^{\mu} \Gamma_{a b}^{d}-\Gamma_{a b}^{\mu} \Gamma_{c d}^{d}\right) \\
& \left.+g^{a b} g^{c d}\left(\Gamma_{a c}^{\mu} \Gamma_{b d}^{\nu}-\Gamma_{a b}^{\mu} \Gamma_{c d}^{\nu}\right)-2 \Lambda g^{\mu \nu}\right] .
\end{aligned}
$$

The Landau-Lifshitz pseudotensor is only a tensor under linear coordinate transformations. To describe the experience of an infalling observer, we evaluate this tensor in the local inertial frame right before the observer crosses the horizon.

Near horizons, we assume a near-Rindler metric of the form $^{12}$

$$
\begin{aligned}
d s^{2} & =-\frac{4 \pi}{\beta}\left(r-r_{H}\right) d t^{2}+\frac{d r^{2}}{\frac{4 \pi}{\beta}\left(r-r_{H}\right)}+r^{2} d x^{i} d x^{i} \\
& =-\frac{\tilde{c} \beta r_{H}}{\pi} d u d v+r_{H}^{2}(1-\tilde{c} u v)^{2} d x^{i} d x^{i},
\end{aligned}
$$

where $\tilde{c}$ is an order-one constant depending on the details of the geometry. With localized perturbations,

$$
T_{u u}=E e^{\frac{2 \pi}{\beta} t_{w}} \frac{\beta}{2 \pi r_{H}^{D-2}} \delta(u) \delta^{(D-2)}\left(x-x_{w}\right),
$$

the metric changes to

$d s^{2}=-\frac{\tilde{c} \beta r_{H}}{\pi} d u[d v-\delta(u) h(x) d u]+r_{H}^{2}(1-\tilde{c} u v)^{2} d x^{i} d x^{i}$.

At large $x-x_{w}$, when the delta function perturbation is a good approximation,

$$
h(x)=\tilde{c} E r_{H} \frac{e^{\frac{2 \pi}{\beta}\left(t_{w}-t_{*}-\frac{\left|x-x_{w}\right|}{v_{B}}\right)}}{\left|x-x_{w}\right|^{\frac{D-3}{2}}},
$$

where $\tilde{c}$ is a dimension-dependent constant.

\footnotetext{
${ }^{12}$ This metric is not exactly the Rindler metric. What we need here is an approximation of horizon metric to first order in $u v$, while in the Rindler metric, the transverse size is a constant.
}

Kruskal coordinates $u, v$ in Eq. (D7) are not local inertial frames at $u=0, v=v_{0}, x=x_{0}$. We do coordinate transformations:

$$
\begin{aligned}
& \tilde{u}=u, \\
& \tilde{v}=v-v_{0}-\frac{\pi r_{H}}{\beta} v_{0}\left(1-\tilde{c} u v_{0}\right)^{2}\left(x-x_{0}\right)^{2}, \\
& \tilde{x}=\left(1-\tilde{c} u v_{0}\right)\left(x-x_{0}\right) .
\end{aligned}
$$

Here $\tilde{u}, \tilde{v}, \tilde{x}$ give the local initial frame right before an observer crosses the horizon at $\tilde{u}=0, \tilde{v}=0, \tilde{x}=0$. In these coordinates, the shockwave metric (D9) becomes ${ }^{13}$

$$
\begin{aligned}
d s^{2}= & -\frac{\tilde{c} \beta r_{H}}{\pi} d \tilde{u}\left[d \tilde{v}-\delta(\tilde{u}) h\left(x_{0}+\frac{\tilde{x}}{1-\tilde{c} \tilde{u} v_{0}}\right) d \tilde{u}\right] \\
& +r_{H}^{2}(1-2 \tilde{c} \tilde{u} \tilde{v}) d \tilde{x}^{2}+\mathcal{O}(\tilde{u}, \tilde{x})^{2} .
\end{aligned}
$$

Evaluating the Landau-Lifshitz pseudotensor (D6) in this frame, we see that, apart from the cosmological constant term,

$$
\begin{aligned}
t_{u u} & =\frac{1}{8 \pi G_{N}} \tilde{c} \delta(u) h(x) \cdot t_{\tilde{u} \tilde{u}} \\
& =\frac{1}{8 \pi G_{N}} c \delta(\tilde{u}) h\left(x_{0}+\frac{\tilde{x}}{1-c \tilde{u} v_{0}}\right) .
\end{aligned}
$$

Assume that we also send in a thermal scale quantum from the right-hand side. To consider the collision energy, we can look at the quantity appearing in the Raychaudhuri equation $-8 \pi G_{N} \frac{\Delta A}{A} \frac{t_{a b} E_{0}^{a} E_{0}^{b}}{\theta^{2}}$. From the definition of the stress-energy tensor $T^{a b}=p^{a} \frac{d x^{b}}{d \tau} \int d \tau \frac{1}{\sqrt{-g}} \delta^{(D)}(x-x(\tau))$, one can verify that ${ }^{14}$

$$
\frac{E_{c}^{2}}{m_{p}^{2}} \sim \frac{r_{H}}{\beta} S^{D-2} 8 \pi G_{N}\left(\frac{-\Delta A}{A}\right) \frac{t_{a b} E_{0}^{a} E_{0}^{b}}{\theta^{2}} .
$$

On the other hand, a direct calculation using Eq. (D11) shows that ${ }^{15}$

$$
-8 \pi G_{N} \frac{\Delta A}{A} \frac{t_{a b} E_{0}^{a} E_{0}^{b}}{\theta^{2}} \sim \frac{h(x)}{v}=\left(\frac{d \tilde{v}^{*}}{d v^{*}}\right)^{-1}-1 .
$$

So the collision energy squared with gravitons is also inversely proportional to the time dilation factor.

\footnotetext{
${ }^{13}$ Note that here we ignore higher-than-first-order terms in $\tilde{u}, \tilde{x}$, but we keep the $\tilde{u} \tilde{v}$ term, because $\tilde{v}$ is not continuous across the horizon.

${ }^{14}$ In fact, one can already see this from the calculations of spherical perturbations in Eqs. (2.12), (D3), and (D5).

${ }^{15}$ The cosmological constant term does not contribute to this.
} 
[1] L. Susskind, Computational complexity and black hole horizons, Fortschr. Phys. 64, 24 (2016).

[2] B. Swingle, Entanglement renormalization and holography, Phys. Rev. D 86, 065007 (2012).

[3] B. Swingle, Constructing holographic spacetimes using entanglement renormalization, arXiv:1209.3304.

[4] T. Hartman and J. Maldacena, Time evolution of entanglement entropy from black hole interiors, J. High Energy Phys. 05 (2013) 014.

[5] D. A. Roberts, D. Stanford, and L. Susskind, Localized shocks, J. High Energy Phys. 03 (2015) 051.

[6] A. R. Brown, D. A. Roberts, L. Susskind, B. Swingle, and Y. Zhao, Complexity, action, and black holes, Phys. Rev. D 93, 086006 (2016).

[7] D. Stanford and L. Susskind, Complexity and shock wave geometries, Phys. Rev. D 90, 126007 (2014).

[8] A. R. Brown, D. A. Roberts, L. Susskind, B. Swingle, and Y. Zhao, Holographic Complexity Equals Bulk Action?, Phys. Rev. Lett. 116, 191301 (2016).

[9] L. Susskind, Entanglement is not enough, Fortschr. Phys. 64, 49 (2016).

[10] L. Susskind, The typical-state paradox: Diagnosing horizons with complexity, Fortschr. Phys. 64, 84 (2016).

[11] Y. Zhao, Complexity, boost symmetry, and firewalls, arXiv:1702.03957.

[12] S. H. Shenker and D. Stanford, Black holes and the butterfly effect, J. High Energy Phys. 03 (2014) 067.

[13] S. H. Shenker and D. Stanford, Multiple shocks, J. High Energy Phys. 12 (2014) 046.
[14] G. 't Hooft, On the quantum structure of a black hole, Nucl. Phys. B256, 727 (1985).

[15] J. M. Maldacena, Eternal black holes in anti-de Sitter, J. High Energy Phys. 04 (2003) 021.

[16] Y. Atia and D. Aharonov, Fast-forwarding of Hamiltonians and exponentially precise measurements, Nat. Commun. 8, 1572 (2017).

[17] L. Susskind and Y. Zhao, Switchbacks and the bridge to nowhere, arXiv:1408.2823.

[18] A. R. Brown, L. Susskind, and Y. Zhao, Quantum complexity and negative curvature, Phys. Rev. D 95, 045010 (2017).

[19] T. Dray and G. 't Hooft, The gravitational shock wave of a massless particle, Nucl. Phys. B253, 173 (1985).

[20] K. Sfetsos, On gravitational shock waves in curved spacetimes, Nucl. Phys. B436, 721 (1995).

[21] J. Maldacena and L. Susskind, Cool horizons for entangled black holes, Fortschr. Phys. 61, 781 (2013).

[22] L. Lehner, R. C. Myers, E. Poisson, and R. D. Sorkin, Gravitational action with null boundaries, Phys. Rev. D 94, 084046 (2016).

[23] L. D. Landau and E. M. Lifschits, The Classical Theory of Fields, Course of Theoretical Physics, Vol. 2 (Pergamon Press, Oxford, 1975).

[24] D. Harlow, The Ryu-Takayanagi formula from quantum error correction, Commun. Math. Phys. 354, 865 (2017).

[25] S. W. Hawking and G. F. R. Ellis, The Large Scale Structure of Space-Time, Cambridge Monographs on Mathematical Physics (Cambridge University Press, Cambridge, England, 2011). 